\title{
HERMITIAN VECTOR BUNDLES AND THE EQUIDISTRIBUTION OF THE ZEROES OF THEIR HOLOMORPHIC SECTIONS
}

BY

\author{
RAOUL BOTT and S. S. CHERN
}

Harvard University, Cambridge, Mass., U.S.A., and University of California, Berkeley, Calif., U.S.A. (1)

\section{Introduction}

At present a great deal is known about the value distribution of systems of meromorphic functions on an open Riemann surface. One has the beautiful results of Picard, E. Borel, Nevanlinna, Ahlfors, H. and J. Weyl and many others to point to. (See [1], [2].) The aim of this paper is to make the initial step towards an $n$-dimensional analogue of this theory.

A natural general setting for the value distribution theory is the following one. We consider a complex $n$-manifold $X$ and a holomorphic vector bundle $E$ over $X$ whose fiber dimension equals the dimension of $X$ and wish to study the zero-sets of holomorphic sections of $E$.

When $X$ is compact (and without boundary) then it is well-known that if the zeroes of any continuous section are counted properly then the algebraic sum of these zero-points is independent of the section and is given by the integral of the $n$th Chern $\left({ }^{2}\right)$ class of $E$ over $X$ : Thus we have

$$
\text { Number of zeroes of } s=\int_{X} c_{n}(E) \text {, }
$$

and this formula is especially meaningful for a holomorphic section because the indexes of all the isolated zeroes of such a section are necessarily positive.

The central question of the value distribution theory is to describe the behavior of the zeroes of holomorphic sections when $X$ is not compact. (For continuous sections there

(1) This work was partially supported by a grant from the National Science Foundation. The second author was a professor of the Miller Institute at the University of California (Berkeley) and received partial support from the Office of Naval Research.

(2) With misgivings on the part of the second author we have adopted a terminology now commonly used. 
are no restrictions in that case, for instance there is always a section which does not vanish at all!)

The main results, all concerned with the case $\operatorname{dim} X=1$, then take the following form. One considers a finite-dimensional "sufficiently ample" subspace $V$ of the space of all holomorphic sections of $E$ and shows that under suitable convexity conditions on $E$ and $X$ "most" of the sections in $V$ vanish the "same number of times". Depending on how "most" and "same number of times" are defined. one gets results of various degrees of delicacy and difficulty. For example, the classical Picard theorem asserts that when $X$ is the Gauss-plane, so that $E$ may be taken as the trivial line bundle $\mathbf{C}$, and $\operatorname{dim} V=2$, then at most 2 sections of $V$ in general position can fail to vanish on $X$. The Borel generalization of this theorem asserts that when $\operatorname{dim} V=n$, then at most $n$ sections in $V$, in general position, can fail to vanish. Here, as throughout, the term general position is used in the following sense: A set of $n$ elements $v_{1}, \ldots, v_{n}$ of a vector-space $V$ is called in general position, if any subset of $k$ elements span a $k$-dimensional subspace of $V$, for $k=1, \ldots, \operatorname{dim} V$.

In the Nevanlinna theory one again deals with $X=\mathbf{C}, \operatorname{dim} V=2$, but now a deficiency index $\delta(s)$ is defined for every $s \in V-0$, which measures the extent to which $s$ behaves unlike the generic section in $V$. In particular $\delta$ has the properties $\delta(\lambda s)=\delta(s)$, if $\lambda \in \mathbf{C}-0$; $0 \leqslant \delta(s) \leqslant 1$; and finally: $\delta(s)=1$ if $s$ does not vanish on $X$. The "first main theorem" may then be interpreted as asserting that $\delta$ considered as a function on the projective space $P_{1}(V)$ of lines in $V$, is equal to 0 almost everywhere. Thus "most" sections in the measure sense behave the same way.

The second main theorem yields the much stronger inequality:

$$
\sum_{i=1}^{m} \delta\left(s_{i}\right) \leqslant 2
$$

valid for any system of sections $s_{i} \in V$ in general position. The Ahlfors generalization deals with the case $\operatorname{dim} V=n$ and again proves among other things that $\delta(s)=0$ nearly everywhere, and that now the inequality

$$
\sum_{i=1}^{m} \delta\left(s_{i}\right) \leqslant n
$$

is valid for any system of $s_{i} \in V$, which are in general position.

Usually these results are stated in terms of maps of $X$ into the Riemann-sphere, (i.e., meromorphic functions) for the Picard and Nevanlinna theory, while the Borel and Ahlfors generalizations deal with maps of $X$ into complex projective spaces of higher dimensions. The transition to our formulation is quite trivial. Indeed consider the evaluation map: $e_{x}: V \rightarrow E_{x}$ which attaches to each section in $V$, the value of $s$ at $x$. By definition, a space of sections $V$ will be "sufficiently ample" if and only if: 
a) $e_{x}: V \rightarrow E_{x}$ is onto for each $x \in X$.

$\beta) \quad V$ contains a section which vanishes to the first order at some point of $X$.

Now let $k(x)$ be the kernel of $e_{x}$. This is then a subspace of a fixed dimension $m=\operatorname{dim} V-\operatorname{dim} E_{x}$, in $V$, so that the assignment $x \rightarrow k(x)$ defines a map $e_{V}: X \rightarrow P_{m}(V)$ of $X$ into the Grassmannian of $m$-dimensional subspaces in $V$.

Now for each $s \in V$, let $z(s)$ be the subvariety of $P_{m}(V)$ consisting of those subspaces which contain $s$. Then, for $s \neq 0, z(s)$ has codimension $n$ in $P_{m}(V)$, and it is clear that the zeroes of $s$ on $X$ correspond precisely to the intersections of $e_{V}(X)$ with $z(s)$ in $P_{m}(V)$.

In particular, when $\operatorname{dim} E_{x}$ is $1, P_{m}(V)$ is just a projective space, and $z(s)$ is a hyperplane, so that we may reformulate our statements in the terms of the number of hyperplanes which the image of $X$ avoids.

Conversely, starting with a map $e: X \rightarrow P_{m}(V)$, one may pull back the quotient bundle of $P_{m}(V)$ (see the end of Section 6 ) to obtain a bundle $E$ over $X$, together with a finite dimensional subspace, $V$, of sections of $E$, for which $e_{V}=e$. Indeed, let $K \subset X \times V$, consist of the subset $(x, v)$ for which $v \in e(x)$. Then $K$ is a sub-bundle of the trivial bundle $X \times V$, and the corresponding quotient bundle, $X \times V / K$ is the desired bundle $E$. The constant sections of $X \times V$ over $X$, then go over into the desired subspace, $V$, of sections of $E$. Thus these two points of view are completely equivalent.

The aim of this paper is to discuss the $n$-dimensional case and we are able to push to an analogue of the first main theorem. Thus we obtain the weak equidistribution in the measure sense only. On the other hand this generalization is not quite immediate and in fact depends on a formula in the theory of characteristic classes, which seems to us of independent interest. To formulate this result we need to recall two facts: Namely 1) That the complex structure on $X$ induces a natural "twisted boundary operator", $d$ c, on the real differential forms, $A(X)$, of $X$, and 2) That a given Hermitian structure on $E$ determines definite representatives, $c_{k}(E) \in A(X), k=1, \ldots, n$, of the Chern classes of $E$. With this understood, we consider a given Hermitian, complex $n$-bundle $E$, over $X$ and its Chern form $c_{n}(E) \in A(X)$. Also let $B^{*}(E)=\{e \in E|0<| e \mid<1\}$ be the subset of vectors in $e$ which are of length greater than 0 and length less than 1 , and set $\pi: B^{*}(E) \rightarrow X$ equal to the natural projection. Then our first and principal result is expressed by the theorem:

THEOR EM I. There exists a real valued form $\varrho$ on $B^{*}(E)$ which is of type $(n-1, n-1)$ and for which

$$
\pi^{*} c_{n}(E)=\frac{d d^{c}}{4 \pi} \varrho
$$

Further if $E$ is non-negative then $\varrho$ may be chosen to be non-negative. 
Remark that $B^{*}(E)$ has the homotopy type of the unit sphere bundle $S(E)$, of $E$, and it is of course well known that $c_{n}(E)$, when lifted to $S(E)$, becomes a boundary. Hence Theorem I refines this result for the complex analytic model $B^{*}(X)$ of $S(E)$.

The method which leads to Theorem I also yields the following auxillary result.

Proposition 1.4. Let $E$ be a complex analytic bundle and let $c(E)$ and $c^{\prime}(E)$ be the Chern forms of $E$ relative to two different Hermitian structures. Then $c(E)-c^{\prime}(E)=d d^{c} \cdot \lambda$ for some $\lambda$.

In other words, if we define $\hat{H}^{k}(X)$ by:

$$
\hat{H}^{k}(X)=A^{k, k}(X) \cap \operatorname{Ker}(d) / d d^{c} A^{k-1, k-1}(X)
$$

then the class in $\hat{H}^{*}(X)=\sum \hat{H}^{k}(X)$ of the Chern form $c(E)$, of $E$ relative to some Hermitian structure on $E$, is independent of that Hermitian structure, so that we may define a "refined Chern class" $\hat{c}(E) \in \hat{H}^{*}(X)$. (Cf. Section 3 for definition of $A^{k, k}(X)$.)

In fact, Theorem I will follow directly from the following Whitney type duality theorem concerning these refined Chern classes:

Proposition 1.5. Let $0 \rightarrow E^{\prime} \rightarrow E \rightarrow E^{\prime \prime} \rightarrow 0$ be an exact sequence of holomorphic vector-bundles over $X$. Then their refined Chern classes satisfy the duality formula:

$$
\hat{c}\left(E^{\prime}\right) \cdot \hat{c}\left(E^{\prime \prime}\right)=\hat{c}(E) .
$$

The formula (1.2) is very pertinant for the whole Nevanlinna theory; for instance in the one-dimensional case, $\varrho$ is just a real valued function on $B^{*}(E)$, and is seen to be minus the logarithmic "height" function:

$$
\varrho(e)=-\log |(e)|^{2}, \quad e \in B^{*}(E) .
$$

Indeed one may roughly express the situation by saying that the first "main inequality" of the Nevanlinna theory is just a twice integrated version of (1.3).

The plan of the paper is as follows: In Section 2 we review the theory of characteristic classes as found in [3], [5]. We then go on to refine this theory for complex analytic Hermitian bundles in Sections 3 to 5 . Section 6 is devoted to a proof of the generalized GaussBonnet theorem which fits into the context of this paper. In Section 7 we define the order function, while in Section 8 we formulate and start to prove the equidistribution theorem. Sections 9 and 10 then complete this proof. Our final section brings a leisurely account of the classical Nevanlinna theorem. This Section 11 is included primarily to show how much more will have to be done before an $n$-dimensional analogue of this delicate theorem is established. 


\section{Curvature and characteristic classes}

In this section $E$ will denote a $C^{\infty}$-bundle over the $C^{\infty}$ manifold $X$. We write $T=T(X)$ for the cotangent bundle of $X$, and $A(X)=\sum A^{i}(X)$ for the graded ring of $C^{\infty}$ complex valued differential forms on $X$. The differential operator on $A(X)$ is denoted by $d$. More generally we write $A(X ; E)$ for the differential forms on $X$ with values in $E$. Thus if $\Gamma(E)$ denotes the $C^{\infty}$ sections of $E$, then $A(X ; E)=A(X) \otimes{ }_{A^{0}(X)} \Gamma(E)$.

The natural pairing from $\Gamma(E) \otimes A_{A^{0}(X)} \Gamma(F)$ to $\Gamma(E \otimes F)\left({ }^{1}\right)$ will often be written simply as multiplication.

Our aim here is to give an elementary and essentially selfcontained review of the geometric theory of characteristic classes, as developed by Chern and Weil. More precisely, we will describe how the curvature of a connection on the vector bundle $E$ can be used to construct closed differential forms on $X$ whose cohomology classes are independent of the connection chosen and therefore furnish topological invariants of the bundle $E$. Of the many definitions of a connection we will use the differential operator one. It leads to the simplest local formulae. We will also thereby avoid the possibly less elementary concept of principal bundles. For a more general account of this theory see [3], [4], [5].

DEFINITION 2.1. A connection on $E$ is a differential operator $D: \Gamma(E) \rightarrow \Gamma\left(T^{*} \otimes E\right)$ which is a derivation in the sense that for any $f \in A^{0}(X)$ :

$$
D(f s)=d f \cdot s+f \cdot D s, \quad s \in \Gamma(E)
$$

Remarks. In general a differential operator from $\Gamma(E)$ to $\Gamma(F)$ is just a C-linear map which decreases supports. If such an operator is also $A^{0}(X)$ linear, then it is induced by a linear map from $E$ to $F$, i.e., by a section of $\operatorname{Hom}(E, F)$. Thus if $D_{1}$ and $D_{2}$ are connections then $D_{1}-D_{2}$ is induced by an element of

$$
\Gamma \operatorname{Hom}\left(E^{*}, T^{*} \otimes E\right)=A^{1}(X ; \operatorname{Hom}(E, E)) .
$$

Suppose now that $E$ is equipped with a definite connection $D$. One may then construct the Chern form of $E$ relative to the connection $D$ in the following manner.

Let $s=\left\{s_{i}\right\}, i=1, \ldots, n$ be a set of sections $\left({ }^{2}\right)$ of $E \mid U$ where $U$ is open in $X$, such that the values $\left\{s_{i}(x)\right\}$ form a base for each $E_{x}$, with $x \in U$. (Such a set $s$ will be called a frame of $E$ over $U$.) In view of (2.2) a formula of the type:

$$
D s_{i}=\sum_{j} \theta_{i j} s_{j}, \quad \theta_{i j} \in A^{1}(U)
$$

(1) The tensor product is over $\mathbf{C}$ unless otherwise indicated.

(2) We will be dealing with smooth sections throughout. 
must then exist and serves to define a matrix of 1 -forms on $U: \theta(s ; D)=\left\|\theta_{i j}\right\|$ - the socalled connection matrix relative to the frame $s$.

In terms of $\theta(s, D)$ one now defines a matrix $K(s, D)=\left\|K_{i j}\right\|$ of 2 -forms on $U$ by the formula: $K_{i j}=d \theta_{i j}-\sum_{\alpha} \theta_{i \alpha} \wedge \theta_{\alpha j}$. In matrix notation:

$$
K(s, D)=d \theta(s, D)-\theta(s, D) \wedge \theta(s, D)
$$

This is the curvature matrix of $D$ relative to the frame $s$. Because even forms commute with one another it makes sense to take the determinant of the matrix $1+i K(s, D) / 2 \pi$ and so to obtain an element $\operatorname{det}\{1+i K(s, D) / 2 \pi\} \in A(U)$.

A priori, this form depends on the frame $s$. However as we will show in a moment, $\operatorname{det}\{1+i K(s, D) / 2 \pi\}$ is actually independent of the frame $s$, and therefore defines a global form, the Chern form of $E$ relative to $D, c(E, D)$ in $A(X)$. More precisely $c(E, D)$ is defined as follows: We cover $X$ by $\left\{U_{\alpha}\right\}$ which admit frames $s^{\alpha}$ over $U_{\alpha}$, and then set $c(E, D) \mid U_{\alpha}=\operatorname{det}\left\{1+i K\left(s^{\alpha}, D\right) / 2 \pi\right\}$. On the overlap these definitions agree because of the asserted independence of our form on the frame $s$.

Consider then two frames $s$ and $s^{\prime}$ over $U$. Then there exist elements $A_{i j} \in A^{0}(U)$ such that $s_{i}^{\prime}=\sum_{j} A_{i j} s_{j}$ and in matrix notation we write simply $s^{\prime}=A s$. From (2.2) it follows that $D s^{\prime}=\{d A+A \theta(s, D)\} s$. Further, by definition, $D s^{\prime}=\theta\left(s^{\prime}, D\right) s^{\prime}$. Hence the connection matrices are related by

$$
d A+A \theta(s, D)=\theta\left(s^{\prime}, D\right) A, \quad s^{\prime}=A s,
$$

from which one directly derives the important formula:

$$
A K(s, D)=K\left(s^{\prime}, D\right) A, \quad s^{\prime}=A s .
$$

This transformation law of the curvature matrix, together with the invariance of the determinant under conjugation now immediately implies the desired independence of our form $\operatorname{det}\{1+i K(s, D) / 2 \pi\}$ on $s$.

Thus we now have defined $c(E, D)$ explicitly and our next aim is to show that $\mathrm{c}(E, D)$ is closed and its cohomology class independent of $D$. For this purpose it is expedient to analyse the above construction a little more carefully, and then to generalize the whole situation.

Note first of all that the transformation law (2.6) is characteristic of the elements of $A(X ; \operatorname{Hom}(E, E))$. Indeed if $\xi \in A^{p}(X ; \operatorname{Hom}(E, E))$ and if $s$ is a frame for $E$ over $U$, then $\xi$ determines a matrix of $p$-forms $\xi(s)=\left\|\xi(s)_{i j}\right\|$ by the formula:

$$
\sum_{j} \xi(s)_{i j} s_{j}=\xi \cdot s_{i}, \quad s=\left\{s_{i}\right\}
$$

and under the substitution $s^{\prime}=A s$, these matrices transform by the law $\xi\left(s^{\prime}\right) A=A \xi(s)$. 
The converse is equally true so that in particular the curvature matrix $K(s, D)$ represents a definite element $K[E, D] \in A^{2}(X ;$ Hom $(E, E))$.

Next we observe that the "determinant construction" really becomes more understandable when formulated in this manner.

We let $M_{n}$ denote the vector-space of $n \times n$ matrices over C. A $k$-linear function $\varphi$ on $M_{n}$ will be called invariant if for all $y \in G L(n, \mathbf{C})$ :

$$
\varphi\left(x_{1}, \ldots, x_{k}\right)=\varphi\left(y x_{1} y^{-1}, y x_{2} y^{-1}, \ldots, y x_{k} y^{-1}\right), \quad x_{\imath} \in M_{n} .
$$

The vector-space of all $k$-linear invariant forms shall be denoted by $I_{k}\left(M_{n}\right)$. Now given $\varphi \in I_{k}\left(M_{n}\right)$ and $U \subset X$, we extend $\varphi$ to a $k$-linear map denoted by $\varphi_{U}$-from $M_{n} \otimes A(U)$ to $A(U)$ by setting:

$$
\varphi_{U}\left(x_{1} w_{1}, x_{2} w_{2}, \ldots, x_{k} w_{k}\right)=\varphi\left(x_{1}, \ldots, x_{k}\right) w_{1} \wedge w_{2} \wedge \ldots \wedge w_{k}, \quad x_{i} \in M_{n}, w_{i} \in A(U) .
$$

With this understood consider $k$ elements $\xi_{i} \in A(X$; Hom $(E, E))$ and let $\varphi \in I_{k}\left(M_{n}\right)$. It is then clear that there is a well-defined form $\varphi\left(\xi_{1}, \ldots, \xi_{k}\right) \in A(X)$, which has the local description:

Given a frame $s$ over $U$, then

$$
\varphi\left(\xi_{1}, \ldots, \xi_{k}\right) \mid U=\varphi_{U}\left\{\xi_{1}(s), \ldots, \xi_{k}(s)\right\}
$$

where the $\xi_{i}(s)$ are the matrices of $\xi_{i}$ relative to $s$ and hence elements of $A(U) \otimes M_{n}$.

We will abbreviate $\varphi(\xi, \xi, \ldots, \xi)$ i.e., the case all $\xi_{i}$ equal, to $\varphi((\xi))$. Now given a connection $D$ on $E$, and a $\varphi \in I_{k}\left(M_{n}\right)$ we have well-determined forms $\varphi((K[E, D]))$ and $\varphi((1+i K[E, D] / 2 \pi))$ in $A(X)$, and our Chern form is clearly of the latter type. Indeed we need only take for $\varphi$ the $n$-multilinear form det on $M_{n}$ obtained by polarizing the polynomial function $x \rightarrow \operatorname{det} x$ on $M_{n}$, to describe the Chern form in the present frame work:

$$
c(E, D)=\operatorname{det}((1+i K[E, D] / 2 \pi)) .
$$

It is now also an easy matter to construct elements $\varphi_{k} \in I_{k}\left(M_{n}\right)$ so that

$$
c(E, D)=\sum \varphi_{k}((K[E, D])) .
$$

In short, the two properties of $c(E, D)$ which we are after will follow from the more conceptual assertion that for any $\varphi \in I_{k}\left(M_{n}\right)$, the form $\varphi((K[E, D]))$ is closed and its homology class independent of $D$.

We will now derive both these properties from the invariance identity (2.8). Note first that differentiation with respect to $y$ leads to the identity

$$
\sum_{=1}^{k} \varphi\left(x_{1}, \ldots,\left[x_{i}, y\right], \ldots x_{k}\right)=0, \quad x_{i}, y \in M_{n}
$$


and conversely-because $G L(n, C)$ is connected-(2.12) implies (2.8). This identity now generalizes in a straight forward manner to the extension of $\varphi_{U}$ and takes the following form in matrix notation. An element $x^{p} \in M_{n} \otimes A^{p}(U)$ (called of $\operatorname{deg} p$ ) is represented by a matrix of $p$-forms. Matrix multiplication therefore gives rise to a pairing $x \otimes y \rightarrow x \wedge y$, of elements of $\operatorname{deg} p$ and $\operatorname{deg} q$ to elements of $\operatorname{deg}(p+q)$. In terms of this multiplication one now defines the bracket $\left[x^{p}, y^{q}\right]$ by the usual formula for graded Lie-algebras:

$$
\left[x^{p}, y^{q}\right]=x \wedge y-(-1)^{p q} y \wedge x .
$$

In this terminology the following invariance law for any $\varphi \in I_{k}\left(M_{n}\right)$ follows directly from (2.9) and (2.10) and (2.12):

$$
\sum(-1)^{q f(\alpha)} \varphi_{U}\left(x_{1}, \ldots,\left[x_{\alpha}, y\right], \ldots, x_{k}\right)=0
$$

whenever the $x_{\alpha}$ and $y$ are homogeneous elements with $q=\operatorname{deg} y$, and $f(\alpha)=\sum_{\beta>\alpha} \operatorname{deg} x_{\beta}$.

From the derivation property of $d$ it follows further that, with the $x_{\alpha}$ as above:

$$
d \varphi_{U}\left(x_{1}, \ldots, x_{k}\right)=\sum(-1)^{g(\alpha)} \varphi_{U}\left(x_{1}, \ldots, d x_{\alpha}, \ldots, x_{k}\right)
$$

where now $g(\alpha)=\sum_{\beta<\alpha} \operatorname{deg} x_{\beta}$.

Proposition 2.16. Let $D$ be a connection for $E$ over $X$, and let $\varphi$ be an invariant form on $M_{n}$. Then $\varphi((K[E, D]))$ is closed.

Proof. This is a local matter. Hence it is sufficient to show that if $s$ is a frame over $U$, then $\varphi_{U}((K)), K=K(s, D)$, is closed on $U$. From (2.14) we obtain

$$
d \varphi_{U}((K))=\sum_{i=1}^{k} \varphi_{U}(K, \ldots, d K, \ldots, K) .
$$

On the other hand the definition, (2.4), of $K(s, D)$ immediately implies the "Bianchiidentity":

$$
d K(s, D)=-[K(s, D), \theta(s, D)] .
$$

Substituting (2.17) and applying (2.14) now yields the desired result.

Proposition 2.18. Let $D_{t}$ be a smooth one parameter family of connections on $E$. Then the function $s \rightarrow d D_{t} s / d t, s \in \Gamma(E)$, is $A^{0}(X)$-linear and hence determines an element $\dot{D}_{t} \in A^{\mathrm{I}}(X ; \operatorname{Hom}(E, E))$.

Further if $\varphi \in I_{k}\left(M_{n}\right)$, then

$$
\int_{a}^{b} \varphi\left(\left(K\left[E, D_{t}\right]\right)\right)=d \int_{a}^{b} \varphi^{\prime}\left(\left(K\left[E, D_{t}\right] ; \dot{D}_{t}\right)\right) d t
$$

where $\varphi^{\prime}((\xi ; \eta))$ stands for $\Sigma_{\alpha} \varphi\left(\xi, \ldots, \eta_{\alpha}, \ldots, \xi\right) .\left({ }^{1}\right)$

(1) $\left.\right|_{a} ^{b} f(t)$ stands for $f(b)-f(a)$. 
Proof. We have $\quad \frac{d}{d t} D_{t} f s=\frac{d}{d t}\left\{f D_{t} s+d f s\right\}=f \frac{d}{d t} D_{t} s$.

This proves the first part, and so in particular that $\varphi^{\prime}\left(\left(K\left[E, D_{t}\right], \dot{D}_{t}\right)\right)$ is a well defined form. For the rest we may work locally relative to a frame $s$ over $U$. It is then easy to see that the matrix of $\dot{D}_{t}$ relative to $s$ is simply the $t$-derivative (denoted by a dot) of the connection from $\theta=\theta\left(s, D_{t}\right)$. Thus $\dot{D}_{t}(s)=\bar{\theta}$. Hence (2.19) will follow from the identity

$$
\dot{\varphi}((K)) \mid U=d \sum_{i} \varphi_{U}(K, \ldots, K, \underset{(i)}{\dot{\theta}}, K, \ldots, K) ; \quad K=K\left(s, D_{t}\right)
$$

Consider now the right hand side (= R.H.S.) of this expression. By (2.15) and (2.17) we obtain:

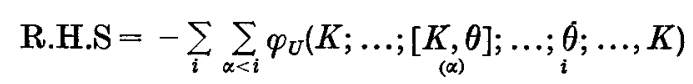

$$
\begin{aligned}
& +\sum_{i} \varphi_{U}(K ; \ldots, K ; d \dot{\theta} ; K, \ldots, K) \\
& +\sum_{i} \sum_{\alpha>i} \varphi_{U}(K, \ldots, \dot{\theta}, \ldots,[K, \theta], K) .
\end{aligned}
$$

Using (2.14) this simplifies to:

$$
\text { R.H.S. }=\sum_{i} \varphi_{U}(K, \ldots, K, d \bar{\theta}-[\dot{\theta} \theta, \theta], K, \ldots, K) .
$$

Finally we recall that $K=d \theta-\theta \wedge \theta$. Hence $\dot{K}=d \dot{\theta}-[\dot{\theta}, \theta]$. Thus the R.H.S. takes the form:

$$
\sum_{j=1}^{k} \varphi_{U}(K, \ldots, \dot{K}, \ldots, K)
$$

which manifestly is just the left hand side $\dot{\varphi}((K))$. Q.E.D.

CoR OLLARY 2.21. The cohomology class of $\varphi((K[E, D]))$ is independent of the connection on $E$.

Indeed if $D_{1}$ and $D_{0}$ are two connections on $E$ then for each $t, D_{t}=t D_{1}+(1-t) D_{0}$ is again a connection on $E$. Hence (2.19) implies the corollary.

Remarks. This concludes our elementary and therefore necessarily rather pedestrian account of the theory of characteristic classes for vector-bundles. A slightly more conceptual path to the same results might run along these lines.

One first notes the following general properties:

(2.22). A pairing of bundles from $E \otimes F$ to $G$ induces a pairing from

$$
A^{p}(X ; E) \otimes A^{q}(X ; F)
$$


to $A^{p+q}(X ; G)$ by combining the above pairing with exterior multiplication. All pairings of this type will be written as a multiplication, i.e., denoted by a dot.

(2.23). If $D$ is a connection for $E$, then the dual bundle $E^{*}$ has a unique connection -also denoted by $D$-which satisfies the equation

$$
d\left\langle s, s^{*}\right\rangle=\left\langle D s, s^{*}\right\rangle+\left\langle s, D s^{*}\right\rangle, \quad s \in \Gamma(E), s^{*} \in \Gamma\left(E^{*}\right) .
$$

(2.24). If $D_{i}$ are connections on $E_{i}, i=1,2$ then the formula

$$
D\left(s_{1} \otimes s_{2}\right)=D_{1} s_{1} \cdot s_{2}+s_{1} \cdot D_{2} s_{2}
$$

defines a connection on $E_{1} \otimes E_{2}, s_{i} \in \Gamma\left(E_{i}\right)$.

(2.25). The connection $D$ on $E$ extends uniquely to an antiderivation of the $A(X)$ module $A(X ; E)$, i.e., so as to satisfy the law:

$$
D(\theta \cdot s)=d \theta \cdot s+(-1)^{p} \theta \cdot D s, \quad \theta \in A^{p}(X), s \in \Gamma(E) .
$$

Now, with these trivialities out of the way, one may argue as follows. First one shows that there is a unique element $K[E, D] \in A^{2}(X$; Hom $(E, E))$ such that

$$
D^{2} s=K[E, D] \cdot s \quad \text { for any } s \in \Gamma(E) \text {. }
$$

One next observes (as we did) that $\varphi \in I_{k}\left(M_{n}\right)$ defines a definite homomorphism

$$
\varphi: \operatorname{Hom}(E, E)^{(k)} \rightarrow 1,
$$

of the $k$ th tensor power of $\operatorname{Hom}(E, E)$ into the trivial bundle, and so induces a map

$$
\varphi: A\left(X, \operatorname{Hom}(E, E)^{(k)}\right) \rightarrow A(X) .
$$

Now, our earlier $\varphi((K[E, D]))$ is defined simply as $\varphi\left\{K[E, D]^{(k)}\right\}$.

One next shows that extension to $A\left(X ; \operatorname{Hom}(E, E)^{(k)}\right)$ of the connection which $D$ induced on $\operatorname{Hom}(E, E)^{k}$, by (2.23) and (2.24) is compatible, with $\varphi$. That is,

$$
d \varphi=\varphi \cdot D
$$

Then the proof of Proposition 2.21 follows directly from this compatibility and the Bianchi-identity: $D K=0$.

\section{Hermitian vector-bundles}

Let $E$ be a vector-bundle over $X$. Then a real-valued function $N: E \rightarrow \mathbf{R}$ is said to define a hermitian structure on $E$-or more briefly to be a norm for $E$-if the restriction of $N$ to any fiber is a Hermitian norm on that fiber. Thus for each $x \in X$, the expression: 


$$
\frac{1}{2}\{N(u+v)-N(u)-N(v)\}+i \frac{1}{2}\{N(u+i v)-N(u)-N(v)\}, \quad u, v \in E_{x}
$$

is to define a positive definite Hermitian form on $E_{x}$. This form will generally be denoted by $\langle u, v\rangle_{x}$, or simply $\langle u, v\rangle$, and upon occasion by $\langle u, v\rangle_{N}$. We of course have $N(u)=\langle u, u\rangle$.

When a complex analytic bundle is endowed with a norm, then the inter-play between these two structures gives rise to several interesting phenomena which will be reviewed in this section.

Recall first of all that on a complex manifold the complex valued differential forms $A(X)$ split into a direct sum $\sum A^{p, q}(X)$ where $A^{p . q}(X)$ is generated over $A^{0}(X)$ by forms of the type $d f_{1} \wedge \ldots \wedge d f_{p} \wedge d f_{p+1} \wedge \ldots \wedge d \tilde{f}_{p+q}$, the $f_{i}$ being local holomorphic functions on $X$. As a consequence $d$ splits into $d^{\prime}+d^{\prime \prime}$ where

$$
d^{\prime}: A^{p, q} \rightarrow A^{p+1, q} \quad \text { and } \quad d^{\prime \prime}: A^{p, q} \rightarrow A^{p, q+1}
$$

These two halves of $d$ are then related by:

$$
d^{\prime 2}=d^{\prime \prime 2}=0, \quad d^{\prime} d^{\prime \prime}+d^{\prime \prime} d^{\prime}=0
$$

If $E$ is a vector-bundle over $X$, then this decomposition of $A(X)$ induces a corresponding decomposition of $A(X ; E)$ into $A^{p, q}(X, E)=A^{p, q}(X) \otimes_{A^{0}(X)} \Gamma(E)$, and hence any connection $D$ on $E$, splits canonically into the sum of

$$
D^{\prime}: \Gamma(E) \rightarrow A^{1,0}(X ; E) \quad \text { and } \quad D^{\prime \prime}: \Gamma(E) \rightarrow A^{0.1}(X ; E) .
$$

With these preliminaries out of the way we come to the first consequence of the simultaneous existence of a holomorphic and Hermitian structure on $E$.

Proposition 3.2. Let $N$ be a Hermitian norm on the analytic bundle $E$, Then $N$ induces a canonical connection $D=D(N)$ on $E$ which is characterized by the two conditions:

(3.3) $D$ preserves the norm $N$.

(3.4) If $s$ is a holomorphic section of $E \mid U$ then $D^{\prime \prime} s=0$ on $U$.

The first condition is expressed by the formula:

$$
d\left\langle s, s^{\prime}\right\rangle=\left\langle D s, s^{\prime}\right\rangle+\left\langle s, D s^{\prime}\right\rangle, \quad s, s^{\prime} \in \Gamma(E),
$$

where we defined $\left\langle s, s^{\prime}\right\rangle$ as the function $\left\langle s, s^{\prime}\right\rangle(x)=\left\langle s(x), s^{\prime}(x)\right\rangle_{x}$ and we have in general set $\left\langle s \otimes \theta, s^{\prime} \otimes \theta^{\prime}\right\rangle$ equal to $\theta \wedge \bar{\theta}^{\prime} \cdot\left\langle s, s^{\prime}\right\rangle$.

The proof of Proposition 3.2 is straightforward. If $s$ is a frame for $E$, over $U$, we write $N(s)$ for the matrix of functions:

$$
N(s)=\left\|\left\langle s_{j}, s_{k}\right\rangle\right\| .
$$

6-652932 Acta mathematica 114. Imprimé le 11 août 1965. 
This is the norm of the frame. Now, let $s$ be a holomorphic frame over $U$, and let $\theta$ be a prospective connection matrix for $E$, relative to $s$. Then (3.5) applied to all the pairs $\left\langle s_{j}, s_{k}\right\rangle$ implies the relation

$$
\theta N+N \bar{\theta}^{t}=d N, \quad N=N(s) .
$$

Hence if $\theta$ is to satisfy this condition, and is also to be of type $(1,0)$ so as to satisfy $(3.4)$, then we must have:

$$
\theta=d^{\prime} N \cdot N^{-1} \quad \text { on } U
$$

Thus there is at most one connection with the properties (3.5) and (3.6).

Conversely, let $s=\left\{s_{i}\right\}$ be a holomorphic frame over $U$, and set $N(s)=\left\|\left\langle s_{i}, s_{j}\right\rangle\right\|$ as before. Then the formula:

$$
D s_{i}=\sum\left(d^{\prime} N \cdot N^{-1}\right)_{i j} s_{j}, \quad N=N(s),
$$

defines a connection over $U$, which is seen to be independent of the holomorphic frame $s$ chosen, and hence induces a global connection $D(N)$ on $E$ which manifestly satisfies the condition of our proposition. The independence of $D$ on $s$ is proved as follows:

Let $s_{1}=A s$ be another holomorphic frame, over $U$. Then $d A=d^{\prime} A$, because $A$ is a holomorphic matrix. Further $N_{1}=N\left(s_{1}\right)=A N A^{t}$. Hence

$$
d^{\prime} N_{1} \cdot N_{1}^{-1}=d A \cdot A^{-1}+A d^{\prime} N \cdot N^{-1} A^{-1}
$$

which shows that the matrices $\theta(s, N)=d^{\prime} N^{\prime} \cdot N^{-1}, N=N(s)$ transform like the connection matrix of a connection.

CoRollary 3.10. Let $E$ be a holomorphic bundle with Hermitian norm $N$, and let $\theta, K$ denote the connection and curvature matrices of $D(N)$ relative to a holomorphic frame over $U$. Then on $U$ one has:

$$
\begin{gathered}
\theta \text { is of type }(1,0), \text { and } d^{\prime} \theta=\theta \wedge \theta . \\
K=d^{\prime \prime} \theta, \text { whence } K \text { is of type }(1,1) \text { and } d^{\prime \prime} \cdot K=0 . \\
d^{\prime} K=-[K, \theta] .
\end{gathered}
$$

Proof. The first line follows directly from $\theta=d^{\prime} N \cdot N^{-1}$, where $N=N(s)$ is the norm of $s$. Indeed $d^{\prime} \theta=d^{\prime} d^{\prime} N \cdot N^{-1}-d^{\prime} N \cdot d^{\prime} N^{-1}$ and $d^{\prime} N^{-1}=-N^{-1} \cdot d^{\prime} N \cdot N^{-1}$. The others are even more straightforward. Note that because $K$ is of type $(1,1)$, the characteristic classes of the connection $D(N)$ always are of type $(p, p)$.

These formulae become especially simple when $E$ is a line bundle. Then a holomorphic frame is simply a nonvanishing holomorphic section $s$, so that, relative to $s, \theta=d^{\prime} \log N(s)$ and $K=d^{\prime \prime} d^{\prime} \log N(s)$. Thus in particular, if $E$ admits a global nonvanishing holomorphic section $s$, then

$$
c_{1}(E)=\frac{i}{2 \pi} d^{\prime \prime} d^{\prime} \log N(s)
$$


The next proposition is a refinement of the earlier homotopy formula (2.19). For simplicity, we will abbreviate $K[E, D(N)]$ to $K[E, N]$.

Proposition 3.15. Consider a smooth family of norms $N_{t}$, on the holomorphic bundle $E$. Then the function $\left(s, s^{\prime}\right) \rightarrow d\left\langle s, s^{\prime}\right\rangle_{N_{t}} / d t$ is Hermitian linear over $A^{0}(X)$ and hence determines a section $L_{t} \in \Gamma$ Hom $(E, E)$, by the formula

$$
\left\langle L_{t} \cdot s, s^{\prime}\right\rangle_{N_{t}}=\frac{d}{d t}\left\langle s, s^{\prime}\right\rangle_{N_{t}}, \quad s, s^{\prime} \in \Gamma(E)
$$

If $\varphi$ is any invariant form in $I_{k}\left(M_{n}\right) ; n=\operatorname{dim} E$, then

$$
\int_{a}^{b} \varphi\left(\left(K\left[E, N_{t}\right]\right)\right)=d^{\prime \prime} d^{\prime} \int_{a}^{b} \varphi^{\prime}\left(\left(K\left[E, N_{t}\right] ; L_{t}\right)\right) d t
$$

where, as before, $\varphi^{\prime}((\xi ; \eta))=\sum_{i} \varphi(\xi, \ldots, \xi, \underset{(i)}{\eta}, \xi, \ldots, \xi)$

Proof. We have

$$
\frac{d}{d t}\left\langle f s, f^{\prime} s^{\prime}\right\rangle=f f^{\prime} \cdot \frac{d}{d t}\left\langle s, s^{\prime}\right\rangle
$$

so that $L$ is well defined. Hence $\varphi^{\prime}\left(\left(K\left[E, N_{t}\right] ; L_{t}\right)\right)$ is a global form and it suffices to check the formula

$$
\frac{d}{d t} \varphi\left(\left(K\left[E, N_{t}\right]\right)\right)=d^{\prime \prime} d^{\prime} \varphi^{\prime}\left(\left(K\left[E, N_{t}\right] ; L_{t}\right)\right)
$$

locally. We therefore choose a holomorphic frame $s=\left\{s_{i}\right\}$ over $U$, and set $N=N_{t}(s)$, $K=K\left(s, D\left(N_{t}\right)\right), \theta=\theta\left(s, D\left(N_{t}\right)\right)$. Then the matrix of $L$ relative to $s$, is easily computed to be $\dot{N} N^{-1}$, the dot denoting the $t$-derivative as before. Let us denote this matrix by $L$ also. Finally, we will abbreviate $\varphi^{\prime}\left(\left(K\left[E, N_{t}\right] ; L_{t}\right)\right) \mid U$ to $\square$. Then

$$
d^{\prime} \square=\sum_{j \neq i} \varphi(K, \ldots,-[K, \theta], \ldots, \underset{(i)}{L}, \ldots, K)+\sum_{j} \varphi\left(K, \ldots, d_{(j)}^{\prime} L, \ldots, K\right)
$$

Applying the invariance identity one obtains

$$
d^{\prime} \square=\sum_{j} \varphi\left(K, \ldots, d^{\prime} L+[L, \theta], \ldots, K\right) .
$$

Finally, one now computes directly from $L=\dot{N} N^{-1}$ and $\theta=d^{\prime} N \cdot N^{-1}$, that

$$
\dot{\theta}=d^{\prime} L+[L, \theta] \text {. }
$$

Hence $d^{\prime} \square$ is the form $\varphi^{\prime}\left(\left(K\left[E, D\left(N_{t}\right)\right], \tilde{D}\left(N_{t}\right)\right)\right)$ of Proposition 2.18 so that (2.19) implies. (3.16). Q.E.D. 
This proposition now directly proves Proposition 1.2 of the Introduction. Indeed if $N_{1}$ and $N_{2}$ are two Hermitian norms on $E$, then $N_{t}=(1-t) N_{1}+t N_{2}$ defines a smooth family between these two Hermitian norms, so that the formula of the proposition becomes a special case of (3.16).

As another direct application we have:

COROLLARY 3.21. Suppose $E$ is an n-dimensional complex vector bundle over $X$, with Hermitian norm $N$. Suppose also that $E$ admits $n$ holomorphic sections which span the fiber at each point. Then the refined Chern classes $\hat{c}_{i}(E)$ are zero for $i>0$ so that:

$$
\hat{c}(E)=1 \text {. }
$$

Proof. Let $s$ be the global frame determined by the sections in question, and define a Hermitian norm on $E$, by setting $N_{1}(s)=$ identity. For this norm $\theta(s), K(s)$ and hence $c_{i}\left(E, N_{1}\right), i>0$ clearly vanish. Q.E.D.

Remark. The deformation $D_{t}=D\left(N_{t}\right)$ induced by the variation of $N_{t}$ is not the linear one encountered earlier. Rather, $D_{t}$ satisfies the differential condition:

$$
\dot{D}_{t}(s)=d^{\prime} L_{t}(s)+\left[L_{t}(s), \theta\left(s, D_{t}\right)\right], \quad s \text { any frame }
$$

In other words $\dot{D}_{t}$ is the $D^{\prime}$-derivative of $L_{t}$, and it is clear that much of the foregoing depends on just the existence of some $L_{t} \in \Gamma \operatorname{Hom}(E, E)$ for which (3.23) is valid.

In the remainder of this section we will formulate a generalization of (3.16) along these lines.

DEFINITION 3.24. A connection $D$ on the holomorphic bundle $E$ over $X$, is called of type $(1,1)$ if:

(3.25) For any holomorphic section $s$ of $E \mid U, D^{\prime \prime} s=0$.

(3.26) The curvature matrix $K(s, D)$ of $D$ relative to a frame $s$ over $U$, are of type $(1,1)$, i.e.,

$$
K[E, D] \in A^{\mathbf{1 . 1}}(X ; \operatorname{Hom}(E, E))
$$

This is then clearly an extension of the class of connections induced by Hermitian norms on $E$.

Next consider a family of connections $D_{t}$ of type $(1,1)$. Such a family will be called bounded by $L_{t} \in A^{0}(X ;$ Hom $(E, E))$ if the relation (3.23) holds between

$$
\dot{D}_{t} \in A^{1}(X ; \operatorname{Hom}(E, E))
$$

and $L_{t}$. Note that the elements of $\Gamma \operatorname{Hom}(E, E)$ may be thought of as defining degree 
zero differential operators on $A(X ; E)$ and on $A(X ; \operatorname{Hom}(E, E))$, the latter action being induced by the composition of endomorphisms. With this understood, the bounding condition (3.23) may quite equivalently be expressed by:

$$
\left[D_{t}^{\prime}, L_{t}\right] s=\dot{D}_{t} s \quad \text { for } s \in \Gamma(E) \text {. }
$$

In any case it is now easy to check that our earlier argument leading to (3.16) also proves the following more general homotopy lemma.

Proposition 3.28. Let $D_{t}$ be a smooth family of connections of type $(1,1)$ on the holomorphic bundle $E$. Suppose further that $D_{t}$ is bounded by $L_{t} \in A^{0}(X ; \operatorname{Hom}(E, E))$. Then for any $\varphi \in I_{k}\left(M_{n}\right), n=\operatorname{dim} E$, we have the relation:

$$
\int_{a}^{b} \varphi\left(\left(K\left[E, D_{t}\right]\right)\right)=d^{\prime \prime} d^{\prime} \int_{a}^{b} \varphi^{\prime}\left(\left(K\left[E, D_{t}\right] ; L_{t}\right)\right) d t
$$

We note in conclusion that if $D_{t}$ is related to $L_{t} \in \Gamma\{\operatorname{Hom}(E, E)\}$ by (3.27), and if $D_{0}$ is of type $(1,1)$, then $D_{t}$ will be of type $(1,1)$ for all $t$. Indeed, $D_{t}$ is of type $(1,1)$ if and only if $\theta=\theta\left(s, D_{t}\right)$ satisfies the two conditions: $\theta_{i j} \in A^{1,0}, d^{\prime} \theta=\theta \wedge \theta$, whenever $s$ is a holomorphis frame.

Differentiating these conditions with respect to time one obtains:

$$
\hat{\theta}_{i j} \in A^{1,0}, \quad d^{\prime} \hat{\theta}=[\dot{\theta}, \theta] .
$$

Now if (3.23) holds then-setting $L_{t}(s)$ equal to $L$-we have $d^{\prime} L+[L, \theta]=\tilde{\theta}$. It follows that $d^{\prime} \hat{\theta}=\left[d^{\prime} L, \theta\right]+\left[L, d^{\prime} \theta\right]$ which by resubstituting (3.23) leads to $d^{\prime} \dot{\theta}=[\dot{\theta}, \theta]$. Thus (3.23) together with $\theta_{i j} \in A^{1,0}$ imply the differentiated identities. Q.E.D.

\section{The duality formula}

Consider an exact sequence of holomorphic vector bundles:

$$
0 \rightarrow E_{I} \rightarrow E \rightarrow E_{1 I} \rightarrow 0
$$

over the base manifold $X$. We wish to prove the duality formula: $\hat{c}(E)=\hat{c}\left(E_{I}\right) \hat{c}\left(E_{I I}\right)$ for the refined Chern classes of these bundles.

For this purpose consider a norm $N$ on $E$. Such a norm then induces norms $N_{I}$ on $E_{I}$ and $N_{I I}$ on $E_{I I}$ in a natural manner: The restriction of $N$ to $E_{I}$ defines $N_{I}$, and the restriction of $N$ to the orthocomplement of $E_{I}$-denoted by $E_{I}^{1}-$ determines $N_{I I}$, via the $C^{\infty}$ isomorphism of $E_{I I}$ and $E_{I}^{\perp}$ induced by (4.1).

Thus (4.1) gives rise to three Chern forms in $A(X): c(E)=c(E, N)$, and $c\left(E_{i}, N_{i}\right)$, 
$i=I, I I$; and the duality formula will be established once we prove the following proposition.

Proposition 4.2. There exists a form $\xi$ in $A(X)$ such that

$$
c(E)-c\left(E_{I}\right) \cdot c\left(E_{I I}\right)=d^{\prime \prime} d^{\prime} \xi
$$

The proof of (4.3) is based upon a specific deformation of the canonical connection $D=D(N)$. To describe this deformation we need certain preliminaries concerning the geometric implications of the exact sequence (4.1).

First we introduce the orthogonal projections

$$
P_{i}: E \rightarrow E_{i}, \quad i=I, I I,\left(^{1}\right)
$$

which this situation naturally defines. These are then elements of $\Gamma \operatorname{Hom}(E, E)$ and therefore-interpreted as degree zero operators, they lead to a decomposition of $D=D(N)$ into four parts:

$$
D=\sum_{i j} P_{i} D P_{j}, \quad i j=I, I I .
$$

LEMMA 4.3. In the decomposition just introduced, $P_{i} D P_{i}$ induces the connection $D\left(N_{i}\right)$ on $E_{i}$, while $P_{i} D P_{j}, i \neq j$, are degree zero operators of type $(1,0)$ and $(0,1)$ respectively:

$$
P_{I I} D^{\prime \prime} P_{I}=0, \quad P_{I} D^{\prime} P_{I I}=0 .
$$

Proof. We first show that $P_{i} D P_{j}, i \neq j$ is $A^{0}(X)$ linear. Consider then $P_{i} D P_{j}(f s)$. Using the derivation properly of $D$ we get $P_{i} D P_{j}(f s)=d f \cdot P_{i} P_{j} s+f P_{i} D P_{j} s$. Hence as $P_{i} P_{j}=0$ if $i \neq j$, it follows that $P_{i} D P_{j}$ is a degree zero operator. We next show that $P_{I I} D^{\prime \prime} P_{I}=0$. This is clearly also a degree zero operator. Hence it is sufficient to show that given $e \in E_{x}$, there exists some section $s$ of $E$ near $x$, such that $s(x)=e$ and $P_{I I} D^{\prime \prime} P_{I} s=0$ at $x$. Now because $E_{I}$ is a holomorphic sub-bundle of $E$ we may choose a holomorphic section $s_{1}$ near $x$ such that the two conditions, $s_{1}(x)=P_{I} e ; P_{I} s_{1}=s_{1}$ (i.e., $s_{1} \in \Gamma\left(E_{l}\right)$ ) hold near $x$. We may also choose a $C^{\infty}$ section $s_{2}$ of $E_{I}^{\frac{1}{1}}$ which satisfies the two conditions $s_{2}(x)=P_{I I} e ; P_{I I} s_{2}=s_{2}$ near $x$. Now setting $s=s_{1}+s_{2}$, we clearly have $s(x)=e$. Further $P_{I} D^{\prime \prime} P_{I}\left(s_{1}+s_{2}\right)=0$ near $x$ because $P_{I} s_{2}=0$ and $D^{\prime \prime} s_{1}=0$ there.

The companion statement of (4.4) now follows directly from the fact that $D$ preserves the inner product: We have $d\left\langle P_{I} s, P_{I I} s^{\prime}\right\rangle=0$ whence

$$
\left\langle P_{I I} D P_{I} s, s^{\prime}\right\rangle=-\left\langle s, P_{I} D P_{I I} s^{\prime}\right\rangle
$$

so that in particular $P_{I I} D^{\prime \prime} P_{I}=0$ implies $P_{I} D^{\prime} P_{I I}=0$.

(1) Here as in what follows we use the natural projection $E_{I}^{1} \rightarrow E_{I I}$ to identify these two bundles. 
It is now quite straightforward to check that the $P_{i} D P_{i}$ interpreted as differential operators on $E_{i}$, satisfy the two conditions which characterize $D\left(N_{i}\right)$. We note that one precisely needs (4.4) to show that the connection induced by $P_{i} D P_{i}$ on $E_{i}$ is of type $(1,0)$ -that is satisfies (3.4).

The deformation which we need for the duality theorem is now defined by:

$$
D_{t}=D+\left(e^{t}-1\right) \delta, \quad \delta=P_{I I} D P_{I}
$$

By our lemma $\delta$ is a degree zero operator and hence $D_{t}$ a connection for every $t \in \mathbf{R}$. We have further:

L смма 4.7. The family $D_{t}$ defined by (4.6) is "bounded" by the element

$$
P_{I} \in \Gamma \operatorname{Hom}(E, E) \text {. }
$$

Proof. We have to show that $\left[D_{t}^{\prime}, P_{I}\right]=\dot{D}_{t}$, or in other words, that

$$
\left[D^{\prime}, P_{I}\right]+\left(e^{t}-1\right)\left[\delta, P_{I}\right]=e^{t} \delta,
$$

where $[A, B]$ stands for the commutator $A B-B A$. Now it is clear that $\left[\delta, P_{I}\right]=\delta$. Hence we just have to show that $P_{I I} D^{\prime} P_{I} \cdot P_{I}-P_{I} \cdot P_{I I} D^{\prime} P_{I}=P_{I I} D P_{I}$ and that follows directly from (4.4). Q.E.D.

We next investigate the curvature form $K\left[E, D_{t}\right]$, and its decompositions according to the $P_{i}$. Using some obvious identifications the formulae take the following form:

LeMma 4.8. Let $P_{i} K\left[E, D_{t}\right] P_{j}$ be denoted by $K_{j i}\left[E, D_{t}\right]$. Then,

$$
\begin{gathered}
K_{I, I}\left[E, D_{t}\right]=K\left[E_{I}, D_{I}\right]-e^{t} \delta^{*} \wedge \delta \\
K_{I I, I I}\left[E, D_{t}\right]=K\left[E_{I I}, D_{I I}\right]-e^{t} \delta \wedge \delta^{*}, \\
K_{I, I I}\left[E, D_{t}\right]=e^{t} K_{I, I I}\left[E, D_{0}\right] ; \quad K_{I I, I}\left[E, D_{t}\right]=K_{I I, I}\left[E, D_{0}\right]
\end{gathered}
$$

where $\delta^{*}$ denotes the adjoint of the form $\delta \in A^{1}(X ;$ Hom $(E, E))$, and hence by $(4.5)$ represents the operator $-P_{I} D P_{I I}$.

These formulae clearly show the pertinence of our deformation $D_{t}$ to the problem at hand. When $t=-\infty$, we see that $K_{I, I I}=0$ and the $K_{i, i}\left[E, D_{t}\right]$ reduces to $K\left[E_{i}, D_{i}\right]$. As the Chern form $c\left(E, D_{t}\right)$ is defined by $\operatorname{det}\left(\left(1+i / 2 \pi K\left[E, D_{t}\right]\right)\right)$ it follows directly that

$$
\lim _{t \rightarrow-\infty} c\left(E, D_{t}\right)=c\left(E_{I}, D_{l}\right) \cdot c\left(E_{I I}, D_{I I}\right)
$$

The proof of Lemma 4.8 is quite straightforward. If one interprets $K$ as the operator $D^{2}$, the terms can be just read off. Alternately one may choose a frame $s=(u, v)$ which is 
naturally suited to the problem-namely of the following type: 1) The frame $s$ is unitary, and its first $k$ components, $u$, span $E_{I}$. (Hence the remaining components- $v-\operatorname{span} E_{I}^{\perp}$.) For such a frame $\theta\left(s, D_{t}\right)$ breaks into blocks $\theta_{i j}\left(s, D_{t}\right)$-corresponding to the operators $P_{j} D P_{i}$ - and then (4.8) follows directly from (2.4).

We have now nearly completed our argument. Indeed, in view of (4.7) and the general homotopy lemma, one has the formula:

$$
\int_{t}^{0} c\left(E, D_{t}\right)=d^{\prime \prime} d^{\prime} \int_{t}^{0} \operatorname{det}^{\prime}\left(\left(1+\varkappa K ; \varkappa P_{I}\right)\right) d t, \quad K=K\left[E, D_{t}\right], \varkappa=i / 2 \pi
$$

valid for all $\left.t \in \mathbf{R} .{ }^{1}\right)$ Hence if we could simply put $t=-\infty$, in (4.13), we would be done. However the integral will not converge in general. In fact, it follows from (4.8) that

$$
K\left[E, D_{t}\right]=K\left[E, D_{-\infty}\right]+e^{t} \square,
$$

where $\square \in A^{2}(X ;$ Hom $(E, E))$ is independent of $t$. Therefore:

$$
\operatorname{det}^{\prime}\left(\left(1+\varkappa K\left[E, D_{t}\right] ; \varkappa P_{I}\right)\right)=\sum_{\alpha=0}^{n-1} a_{\alpha} e^{\alpha t}, \quad a_{\alpha} \in A(X)
$$

with

$$
a_{0}=\operatorname{det}^{\prime}\left(\left(1+\varkappa K\left[E, D_{-\infty}\right] ; \varkappa P_{I}\right)\right) \text {. }
$$

Hence the integral will converge only if $a_{0}=0$, and that is generally not the case. Note however that by (4.7) $a_{0}$ can be re-expressed as

$$
\operatorname{det}^{\prime}\left(\left(1+x K\left[E_{I}, D_{I}\right] ; \varkappa 1\right)\right) \cdot \operatorname{det}\left(\left(\varkappa K\left[E_{I I}, D_{I I}\right]\right)\right) .
$$

It then follows easily that $a_{0}$ is a linear combination of the Chern forms $c_{\alpha}\left(E_{I}, N_{I}\right)$ multiplied by $c_{n-k}\left(E_{I I}, N_{I I}\right)$ :

$$
a_{0}=\left\{\sum_{\alpha=0}^{k}(n-k) c_{\alpha}\left(E_{I}\right)\right\} c_{n-k}\left(E_{I I}\right)
$$

In any case $a_{0}$ will be a closed form.

Hence (4.13) will remain valid even when $a_{0}$ is deleted from under the integral sign. But once this is done one may clearly integrate and pass to the limit $t=-\infty$, in (4.13) to obtain:

$$
c(E)-c\left(E_{I}\right) \cdot c\left(E_{I I}\right)=d^{\prime \prime} d^{\prime}\left\{\sum_{\alpha=1}^{n} \alpha^{-1} \cdot a_{\alpha}\right\} ; \quad a_{\alpha} \text { as in (4.15). }
$$

This then completes the proof of the duality formula, and also gives us the explicit form $\sum_{\alpha=1}^{n} \alpha^{-1} \cdot a_{\alpha}$ for $\xi$. In the proof of Theorem I we need to compute the highest component of $\xi$ for the case, $\operatorname{dim} E_{I}=1$. Thus we want to expand $x^{n} \operatorname{det}\left(\left(K\left[E, D_{-\infty}\right]+e^{t} \square ; P\right)\right)$

(') $\operatorname{det}^{\prime}$ is the function denoted by $\varphi^{\prime}$, with $\varphi=\operatorname{det}$, in Section 3; see also (4.19) below. 
under that assumption. Again using (4.7) we see that because $\operatorname{dim} E_{I}=1$ this expression reduces to $x^{n} \operatorname{det}\left(\left(K\left[E_{I I}, N_{I I}\right]+e^{t} \square_{I I}\right)\right)$ where $\square_{I I}=K_{I I, I I}[E, D]-K\left[E_{I I}, N_{I I}\right]$. Hence if we define $\operatorname{det}^{\alpha}((\xi ; \eta))$ by the identity

$$
\operatorname{det}((\xi+\lambda \eta))=\sum \lambda^{\alpha} \operatorname{det}^{\alpha}((\xi ; \eta))
$$

then the coefficients $a_{\alpha}$ which we are after, are given by $x^{n} \operatorname{det}^{\alpha}\left(K\left[E_{I I}, N_{I I}\right] ; \square_{I I}\right)$. We record this fact for later reference:

Proposition 4.20. Let $0 \rightarrow E_{I} \rightarrow E \rightarrow E_{I I} \rightarrow 0$ be an exact sequence of holomorphic vector bundles, and let $c(E)$, and $c\left(E_{i}\right), i=I, I I$ denote the Chern forms induced by a norm $N$ on $E$. Then if $\operatorname{dim} E_{I}=1$,

$$
c_{n}(E)-c_{1}\left(E_{I}\right) \cdot c_{n-1}\left(E_{I I}\right)=\varkappa d^{\prime \prime} d^{\prime}\left\{\sum_{\alpha>0} \alpha^{-1} \operatorname{det}^{\alpha}\left(\left(\Omega\left[E_{I I}\right] ;-\Delta_{I I}[E]\right)\right)\right\}
$$

where $\Omega\left[E_{I I}\right]=\varkappa K\left[E_{I I}, N_{I I}\right], \Omega_{I I}[E]=\varkappa K_{I I, I I}[E ; N]$, and $-\Delta_{I I}=\Omega_{I I}[E]-\Omega\left[E_{I I}\right]$. Hence if $E_{I}$ admits a nonvanishing holomorphic section $s$, then by 3.15 ,

$$
c_{n}(E)=\varkappa d^{\prime \prime} d^{\prime}\left\{\log N(s) \cdot c_{n-1}\left(E_{I I}\right)+\sum_{\alpha>0} \alpha^{-1} \cdot \operatorname{det}^{\alpha}\left(\left(\Omega\left[E_{I I}\right],-\Delta_{I I}[E]\right)\right)\right\}
$$

Note that aside from the positivity assertion, (4.22) proves Theorem I. Indeed consider the projection $\pi_{1}: E \rightarrow X$. The identity map of $E$ into itself, then induces a holomorphic section $s$ of $\pi_{1}^{*}(E)$ over $E$, which vanishes only on the zero section $X \subset E$. Hence if $\pi: B^{*}(E) \rightarrow X$ is the restriction of $\pi_{1}$ to the subset $B^{*}(E)=\{e \in E \mid 0<N(e)<1\}$ of $E$ then the section $s$ of $\pi^{*} E=\pi_{1}^{*}(E) \mid B^{*}(E)$ does not vanish. We may therefore apply (4.22) to $\pi^{*}(E)$ and so obtain a formula of the type $c_{n}\left\{\pi^{*}(E)\right\}=x d^{\prime \prime} d^{\prime} \xi$. Now by the obvious functioriality of the Chern forms relative to holomorphic isomorphisms, $c_{n}\left\{\pi^{*}(E)\right\}=\pi^{*} c_{n}(E)$, so that we are done. In the next section we will discuss the positivity of the $\xi$ given by (4.22). Let us close this one with a direct consequence of the duality formula which generalizes (4.22) but in a less specific fashion.

COR OLLARY. Let E be a holomorphic bundle over $E$, which admits k linearly independent holomorphic sections. Then

$$
\hat{c}_{i}(E)=0 \text { for } i>n-k .
$$

Proof. Let $E_{I}$ be the bundle spanned by the sections. Then by $(3.22) \hat{c}\left(E_{I}\right)=1$. Hence by the duality formula $\hat{c}(E)=\hat{c}\left(E / E_{I}\right)$ and the bundle $E / E_{I}$ has dimension $(n-k)$. Q.E.D. 


\section{Remarks on Positivity. The proof of Theorem I completed}

As we have seen (4.22) already proves the combinatorial aspects of Theorem I and it remains only to discuss the "sign" of the $\xi$ there constructed.

We recall first of all that $A^{p, p}(X)$ contains a well determined convex cone of positive $(\geqslant 0)$ elements. By definition a form $\Omega$ is in the cone-noted by $\Omega \geqslant 0$-if and only if there exist forms $\theta_{\alpha} \in A^{p .0}(X)$ such that

$$
\Omega=i^{p^{2}} \sum_{\alpha} \theta_{\alpha} \wedge \bar{\theta}_{x}
$$

We may extend this definition to matrix valued forms in the following fashion:

DEFINITION 5.1. Let $\Omega$ be an $n \times n$ matrix of forms of type $(p, p)$. Then $\Omega$ is positive, if there exist $n \times m$ matrices $N_{r}$, of type $(p, 0)$ such that

$$
\Omega=i^{p^{s}} \sum_{r} N^{r} \wedge \bar{N}_{r}^{t}
$$

Note that if $A$ is any nonsingular $n \times n$ matrix of functions, then $\Omega$ is positive with $A \Omega \bar{A}^{t}$. This enables us to define positivity in $A^{p, p}(X ;$ Hom $(E, E))$ for any bundle $E$ with a Hermitian structure. Namely if $\xi \in A^{D . p}(X$; Hom $(E, E))$ we define $\xi \geqslant 0$ to mean that the matrix of $\xi$ relative to a unitary frame $s$, be positive: $\xi(s) \geqslant 0$. As unitary frames are related by unitary transformations-for which therefore $\bar{A}^{t}=\mathrm{A}^{-1}$, 一 this concept is thereby well defined.

Hence in particular, if $E$ is a holomorphic bundle with Hermitian norm $N$-then it makes sense to ask whether the "real curvature form" $\Omega[E, N]=\varkappa K[E ; N]$ is positive or not.

To simplify the notation we will, in the sequel, call such a holomorphic bundle with a given Hermitian norm simply a Hermitian bundle, denote it by a single letter $E$, and write $K[E]$, $\Omega[E], c(E)$ etc., instead of $K[E ; N], \Omega[E, N], c(E)$ etc. Such a bundle is called positive if $\Omega[E] \geqslant 0$.

That these notions of positivity on the form and the vector-bundle level are compatible follows readily from the following lemma:

LEMMA 5.3. Let $E$ be a Hermitian bundle of dimension $n$, and let $\xi_{r}$ be positive elements of $A^{p, p}(X ; \operatorname{Hom}(E, E)) ; r=1, \ldots, n$. Then if $p$ is odd $\operatorname{det}\left(\xi_{1}, \ldots, \xi_{n}\right) \in A^{p n, p n}(X)$ is positive.

Proof. We may find forms $N_{i \alpha}^{r} \in A^{p .0}(U)$, so that with respect to some unitary frame $s$ over $U$,

$$
\xi_{r}(s)=i^{p^{2}} \sum_{\alpha_{r}} N_{i \alpha_{r}}^{r} \bar{N}_{j \alpha_{r}}^{r}, \quad 1 \leqslant \alpha_{r} \leqslant \beta_{r}
$$

Hence $\operatorname{det}\left(\xi_{1}, \ldots, \xi_{n}\right)$ is given by the sum: 


$$
i^{p^{2} \cdot n}\left(n !^{-1}\right) \sum_{\sigma, \tau, \alpha}(-1)^{\sigma} N_{1 \alpha_{\tau(1)}^{\tau(1)}}^{N_{\sigma(1) \alpha_{\tau(1)}}^{\tau(1)}} \ldots N_{n \alpha_{\tau(n)}(n)}^{\tau(n)} \bar{N}_{\sigma(n) \alpha_{\tau(n)}(n)}^{(n)}
$$

where $\sigma$ and $\tau$ vary over the group of permutations and $\alpha$ denotes independent summation over the $\alpha_{i}{ }^{2} \mathrm{~s}$.

If we now take all the barred terms to the right and reorder them in ascending order according to their first lower index then, because $p$ is odd $(-1)^{\sigma}$ cancels out and this expression is seen to take the form:

$$
(n !)^{-1} i^{p^{2} n} \cdot i^{p^{2}\left(n^{2}-n\right)} \sum_{\lambda_{, \tau, \alpha}} N_{1 \cdot}^{\tau(1)} \ldots N_{n}^{\tau(1)} \cdot \bar{N}_{1 .}^{\lambda(1)} \ldots \bar{N}_{1}^{\lambda(n)}
$$

where $\lambda=\tau \circ \sigma^{-1}$ and we have denoted the appropriate $\alpha$-index by a dot. Hence

$$
\operatorname{det}\left(\xi_{1}, \ldots, \xi_{n}\right)=(n !)^{-1} i^{(p n)^{\alpha}} \sum_{\alpha} \theta_{\alpha} \wedge \bar{\theta}_{\alpha}
$$

where $\theta_{\alpha}=\sum_{\lambda} N_{1}^{\lambda(1)} N_{2}^{\lambda(2)} \ldots N_{n}^{\lambda(n)}$ and therefore clearly $\geqslant 0$. Q.E.D.

As an immediate corollary we have:

(5.4) The forms $\operatorname{det}^{\alpha}((\xi ; \eta))$ are positive if $\xi, \eta \in A^{1.1}(X ; \operatorname{Hom}(E, E))$ are positive. Further if $\xi \geqslant \xi^{\prime} \geqslant 0, \eta \geqslant \eta^{\prime} \geqslant 0$ then $\operatorname{det}^{\alpha}((\xi, \eta)) \geqslant \operatorname{det}^{\alpha}\left(\left(\xi^{\prime} ; \eta^{\prime}\right)\right)$.

Indeed, $\operatorname{det}^{\alpha}(\xi, \eta)$ is just the sum $\sum \operatorname{det}\left(\xi_{1}, \ldots, \xi_{n}\right)$ where $\alpha$ of the $\xi_{i}$ are set equal to $\eta$ and the remaining ones are equal to $\xi$. In particular then, we have:

$$
\Omega(E) \geqslant 0 \Rightarrow c(E) \geqslant 0 \text {. }
$$

When applied to the exact sequence of Hermitian vector bundles (4.1):

$$
0 \rightarrow E_{1} \rightarrow E \rightarrow E_{11} \rightarrow 0
$$

our lemma yields the following inequalities: In the notation of that section, define $\Omega_{i}[E]$, $i=I, I I$, to be the form $\varkappa P_{i} K[E] P_{i}$ interpreted as a section of $A^{2}\left(X ; \operatorname{Hom}\left(E_{i}, E_{i}\right)\right)$. Then in view of (4.4) and (4.8) we immediately obtain the inequalities:

$$
\begin{gathered}
\Delta_{I}(E)=\Omega\left[E_{I}\right]-\Omega_{I}[E] \leqslant 0 \\
\Delta_{I I}(E)=\Omega\left[E_{I I}\right]-\Omega_{I I}[E] \geqslant 0 .
\end{gathered}
$$

Put differently, sub-bundles are less positive and quotient bundles more positive, than the bundle itself.

We next return to the formula $(4.22)$ :

$$
c_{n}(E)=\varkappa d^{\prime \prime} d^{\prime}\left\{\log N(s) \cdot c_{n-1}\left(E_{I I}\right)+\xi\right\}
$$

where

$$
\xi=\sum_{\alpha>0} \alpha^{-1} \operatorname{det}^{\alpha}\left(\left(\Omega\left[E_{I I}\right] ; \Omega_{H}[E]-\Omega\left[E_{I I}\right]\right)\right) \text {. }
$$


Assume now that $\Omega[E] \geqslant 0$. Then $\Omega_{I I}[E] \geqslant 0$ and hence by (5.6) $\Omega\left[E_{I I}\right] \geqslant \Delta_{I I}[E] \geqslant 0$. From this it follows that $c_{n-1}\left(E_{I I}\right) \geqslant 0$. The form $\xi$ can be written as

$$
\sum_{\alpha>0} \alpha^{-1}(-1)^{\alpha} \operatorname{det}^{\alpha}\left\{\Omega\left[E_{I I}\right] ; \Delta_{I I}(E)\right\}
$$

Hence $\xi$ is an alternating sum of positive terms and therefore neither positive or negative. However, this is not serious. In fact we can add to $\xi$ a closed form $\xi_{0}$ so as to make $\xi+\xi_{0} \geqslant 0$ and $\xi-\xi_{0} \leqslant 0$. This is done as follows: Let

$$
\xi_{0}=\sum_{\alpha>0} \alpha^{-1} \operatorname{det}^{\alpha}\left(\left(\Omega\left[E_{1 l}\right] ; \Omega\left[E_{I I}\right]\right)\right)
$$

Then by the definition of $\operatorname{det}^{\alpha}, \xi_{0}=\sum \alpha^{-1}\left(\begin{array}{l}n \\ z\end{array}\right) c_{n-1}\left(E_{I I}\right)$, and hence is a closed form. Further note that in view of (5.4), we have

$$
\operatorname{det}^{\alpha}\left(\left(\Omega\left[E_{I I}\right] ; \Omega\left[E_{I I}\right]\right)\right) \geqslant \operatorname{det}^{\alpha}\left(\left(\Omega\left[E_{I I}\right] ; \Delta_{I I}(E)\right)\right),
$$

and so our assertion concerning $\xi_{0}$ is correct.

We next replace $\xi$ by $\xi-\xi_{0}$ in (4.1) and use the definition

$$
d^{c}=i\left(d^{\prime \prime}-d^{\prime}\right)
$$

The formula (4.7) then takes the form:

$$
c_{n}(E)=\frac{d d^{c}}{4 \pi}\left\{\log N^{-1}(s) c_{n-1}\left(E_{I I}\right)+\left(\xi_{0}-\xi\right)\right\}
$$

with the bracketed term $\geqslant 0$ wherever $\log N^{-1}(s)>0$, i.e., wherever $N(s)<1$. Applied to $B^{*}(E)$, this formula therefore precisely proves Theorem $\mathrm{I}$.

\section{The relative Gauss Bonnet theorem}

We already remarked in the introduction that the first main inequality of the Nevanlinna theory may be thought of as a twice integrated version of the formula (1.2) in Theorem I. The first integral of (1.2) leads to the generalized theorem of Gauss-Bonnet (for the complex case) and so serves to give a geometric interpretation of the Chern classes $c_{i}(E)$.

In this section we will, for the sake of completeness, briefly derive this development. The situation we wish to study is the following one: let $E$ be a holomorphic $n$-bundle with a Hermitian norm $N$, over the complex $n$-manifold $X$ with boundary $\partial X=Y$, and assume that $s_{y}$ is a nonvanishing section of $E$ over $Y$. The question now arises when $s_{y}$ may be extended to all of $X$ without vanishing, and Theorem $I$, in the explicit form given by (4.22) may be interpreted as giving an answer to this question. 
Indeed, let $E_{0} \subset E$, be the subset $\{e \mid N(e)>0\}$ complementary to the zero-section in $E$, and let $\pi_{0}: E_{0} \rightarrow E$ be the projection. As we already remarked, the identity inclusion $E_{0} \rightarrow E$ then induces a nonvanishing section $s_{I}$ of $\pi_{0}^{-1}(E)$ over $E_{0}$, so that the formula (5.10) gives rise to a definite form $\varrho$ over $E_{0}$, for which

$$
\frac{d d^{c}}{4 \pi} \varrho=\pi_{0}^{*} c_{n}(E)
$$

At this stage we will actually only need the form

$$
\eta(E)=\frac{d^{c}}{4 \pi} \varrho
$$

for which we therefore clearly have the identity

$$
\pi_{0}^{*} c_{n}(E)=d \eta(E)
$$

In terms of this form, the answer to our question is given by the following proposition.

Proposition 6.2. The section $s_{y}$ of $E_{0} \mid Y$ may be extended to all of $E_{0}$ if and only if

$$
\int_{X} c_{n}(E)-\int_{X} s_{y}^{*} \eta(E)=0 .
$$

The proof of this proposition follows directly from quite elementary obstruction theory, once it is established that the expression $v\left(X ; Y ; s_{y}\right)=\int_{X} c_{n}(E)-\int_{Y} s_{y}^{*} \eta(E)$, always measures the number of times any extension of $s_{y}$ to $X$ has to vanish. To be more precise we need to recall the topological definition of the order of vanishing of a section $s$ of $E$ at a point $p$ which is an isolated zero of $s$. This is an integer, denoted by zero $(s ; p)$, which is defined as follows:

Let $B_{\varepsilon}$ be a disc of radius $\varepsilon>0$ about $p$, relative to local coordinates centered at $p$. Also, let $\varphi_{p}: E \mid B_{\delta} \rightarrow E_{p}$, be a trivialization of $E$ over $B_{\delta}$, i.e., a retraction of $E \mid B_{\delta}$ onto $E_{p}$ which is an isomorphism on each fiber. For small enough $\varepsilon_{0}$, the map $\varphi_{p} \circ s$ then maps $\partial B_{\varepsilon}$ into $E_{p}-0$, for all $0<\varepsilon<\varepsilon_{0} \leqslant \delta$. The degree of this map is by definition the number zero $(s ; p)$ :

$$
\operatorname{zero}(s ; p)=\operatorname{deg}\left(p_{p} \circ s\right) \quad \text { on } \partial B_{\varepsilon}
$$

(The orientation of $B_{\varepsilon}$ is taken to be the one given by positive forms; similarly we orient the unit disc of $E_{p}$ by the positive forms. There are canonical induced orientations on $\partial B_{\varepsilon}$, and the unit sphere, $S\left(E_{p}\right)$, in $E_{p}$. Using the retraction $E_{p}-0 \rightarrow S\left(E_{p}\right)$ this class serves to orient $E_{p}-0$, so that $\operatorname{deg}\left(\varphi_{p} \circ s\right)$ is well-defined.)

With this understood, (6.2) becomes an easy consequence of the following theorem. 
Proposition 6.4. Let $s$ be a smooth section of $E$ with the following properties:

«) $s \neq 0$ on $\partial X=Y$

B) $s$ has isolated zeroes only.

Under these circumstances one has the formula:

$$
\sum \operatorname{zero}(p ; s)=\int_{X} c_{n}(E)-\int(s \mid Y)^{*} \eta(E)
$$

where $p$ ranges over the set of zeroes $p_{i}, i=1, \ldots, m$ of $s$.

Proof. We first derive (6.5) from the following proposition:

Proposition 6.6. Let $j_{p}: S\left(E_{p}\right) \rightarrow E_{0}$ be the inclusion of the unit sphere of $E_{p}$, into $E_{0}$. Then,

$$
j_{p}^{*} \eta(E)=- \text { the orientation class of } S\left(E_{p}\right)
$$

Granted (6.7) we proceed as follows.

Let $X_{\varepsilon}$ be obtained from $X$ by removing the interiors of little dises $B_{\varepsilon}^{i}$ of radius $\varepsilon$ about $p_{i}$ from $X$. Then there is a $\delta>0$ so that $s$ will not vanish on $X_{\varepsilon}$ for $0<\varepsilon \leqslant \delta$. Also choose trivializations $\varphi_{i}$ of $E \mid B_{\delta}^{i}$. We then have

$$
\int_{X_{\varepsilon}} c_{n}(E)=\int_{X_{\varepsilon}} s^{*} \pi_{0}^{*} c_{n}(E)
$$

because $s$ is a section of $E_{0}$ over $X_{\varepsilon}^{\prime}$. Now by Stokes formula it follows that

$$
\int_{X_{\varepsilon}^{-}} c_{n}(E)=\int_{\partial X_{\varepsilon}} \eta(E)=\int_{Y} s^{*} \eta(E)-\sum \int_{\partial B_{i}^{\varepsilon}} s^{*} \eta(E) .
$$

Using the $\varphi_{i}$ it is now clear that $\int_{\partial B_{i}^{\varepsilon}} s^{*} \eta(E)$ is approximately $\int_{\partial B_{i}^{\varepsilon}} \varphi_{i}^{*} j_{p_{i}}^{*} \eta(E)$ when $\varepsilon$ is small. Hence by $(6.7)-\int_{\partial B_{i}^{\varepsilon}} s^{*} \eta(E) \rightarrow$ zero $\left(s, p_{i}\right)$. Thus $(6.8)$ goes over directly into (6.5) as $\varepsilon \rightarrow 0$. Q.E.D.

It is Proposition 6.6 which therefore lies at the center of these formulae. To prove it one may explicitly integrate the form described by (5.10). Alternatively one may apply the argument we just gave in reverse, to a situation where (6.5) can be established by some other means. We will follow the second alternative because many of the concepts which are needed for this special example will also be used later. Note finally, that because of the functorial definition of $\eta(E) \quad j_{p}^{*} \eta(E)$ is a well determined form on $S_{2 n-1}$ modulo only unitary transformations of that sphere. In short, to prove (6.7) it will be sufficient to find an example of a Hermitian bundle $E$ over the complex manifold $X$ with $\partial X=\varnothing$ together with a section $s$ of $E$, such that: 
a) $s$ has a single isolated zero at $p \in X$, with $\operatorname{zero}(s, p)=1$

B) $\int_{x} c_{n}(E)=1$.

As we will now show, an example of this type is furnished by the complex projective space and the "quotient bundle" over it.

Let then $V$ be a fixed complex vector space, of $\operatorname{dim}(n+1)$, and let $P_{1}(V)$ be the projective space of 1 -dimensional subspaces of $V$. (Note that $\operatorname{dim}_{C} P_{1}(V)=n$.)

Over $P_{1}(V)$ we have the canonical exact sequence

$$
0 \rightarrow S_{1}(V) \rightarrow T_{1}(V) \rightarrow Q_{1}(V) \rightarrow 0
$$

of holomorphic vector-bundles, defined in the following "tautologous" manner.

(6.10) $T_{1}(V)$ is the product bundle $P_{1}(V) \times V$ over $P_{1}(V)$

(6.11) $S_{1}(V)$ is the subset of $T(V)$ consisting of pairs $(l, v)$-where $l \in P_{1}(V)$ is a line in $V$, and $v \in V$ - for which $v \in l$.

(6.12) $Q_{1}(V)$ is the quotient bundle $T_{1}(V) / S_{1}(V)$.

The bundle $Q_{1}(V)$ over $P_{1}(V)$ is the one we called the quotient bundle of the projective space $P_{1}(V)$. Note that each $v \in V$, determines a holomorphic section $s_{v}$ of $Q_{1}(V)$, defined by the projection of the constant section: $x \rightarrow(x, v), x \in P_{1}(V)$, of $T_{1}(V)$ into $Q_{1}(V)$. Clearly, if $v \neq 0$ then $s_{v}$ vanishes at only one point $l \in P_{1}(V)$ namely at the subspace, [v], generated by $v$. Further it is not hard to see that $\operatorname{zero}\left[s_{v},[v]\right]=1$. Thus $\alpha$ ) is satisfied.

To check the condition $\beta$ ) we need a hermitian structure on $T_{1}(V)$ which we of course take to be the trivial one induced by a hermitian structure on $V$. Thus the curvature form of $T_{1}(V)$ is equal to zero. Hence by the inequality (5.7) we note that $\Omega\left\{Q_{1}(V)\right\} \geqslant 0$. Thus in any case $\int_{Q_{1}(V)} c_{n}\left\{Q_{1}(V)\right\} \geqslant 0$. Hence it will be sufficient to show that $c_{n}\left\{Q_{1}(V)\right\}$ is an orientation class for $P_{\mathbf{1}}(V)$ to establish $\beta$ ).

Consider the case $n=1$, first. Let $v_{1}, v_{2}$ be an orthonormal base for $V$ and let $z \rightarrow\left[v_{1}+z v_{2}\right]$ be a local parameter near $\left[v_{1}\right]$. Also let $s_{1}$ be the section $s_{v_{1}}$, which is therefore holomorphic and $\neq 0$ on $P_{1}(V)-\left[v_{1}\right]$. Hence on this set

$$
c_{1}\{Q(V)\}=\frac{d d^{c}}{4 \pi} \ln \left|s_{1}\right|^{-1}
$$

where $\left|s_{1}\right|(l)$ is the norm of the section at $l$. Thus near $\left[v_{1}\right]$ we have,

$$
\left|s_{1}\right|^{2}\left(\left[v_{1}+z v_{2}\right]\right)=|z|^{2} /\left(1+|z|^{2}\right) .
$$

It follows, again by Stokes, that if $B_{\varepsilon}=(|z|<\varepsilon)$ then 


$$
\int_{P_{1}(V)} c_{1}\{Q(V)\}=\lim _{\varepsilon \rightarrow 0}-\int_{\partial B_{\varepsilon}} \frac{d^{c}}{4 \pi}\left[\ln |z|^{2}+\ln \left(1+|z|^{2}\right)\right]
$$

Clearly the second term tends to zero, while the first tends to +1 , as is seen directly if we write $z=r e^{i \theta}, \log z=\log r+i \theta$ and recall that $d^{c}=i d^{\prime \prime}-i d^{\prime}$. Thus $\beta$ ) is true for $n=1$.

To get $\beta$ ) in general one may use the Whitney duality formula. In the present instance this formula yields:

$$
c\left\{S_{1}(V)\right\} \cdot c\left\{Q_{1}(V)\right\}=c\left\{T_{1}(V)\right\}=1
$$

Thus $c_{n}\{Q(V)\}=\left[-c_{1}\left\{S_{1}(V)\right\}\right]^{n}$. For $n=1$, this implies that $c_{1}\left\{S_{1}\left(V_{2}\right)\right\}$ is an orientation class of $P_{1}\left(V_{2}\right)$. Now under the inclusion $V_{2} \rightarrow V, S_{1}(V)$ clearly restricts to $S_{1}\left(V_{2}\right)$. Hence $\left.c_{1} S_{1}(V)\right\}$ restricts to an orientation class of $P_{1}\left(V_{2}\right)$. But then $c_{1}\left\{S_{1}(V)\right\}$ must generate $H^{2}(X ; Z), X=P_{1}(V)$ and hence $(-1)^{n} c_{1}\left\{S_{1}(V)\right\}$ must be an orientation class for $P_{1}(V)$ in general. Q.E.D.

An important corollary of $(6.5)$ is the following interpretation of $c_{n}(E)$ :

COROLLARY 6.13. Let $E$ be a holomorphic n-bundle over the complex n-manifold $X$, and let $s: X \rightarrow E$ be a smooth section of $E$, which is $\neq 0$ on $\partial X$, and which is transversal to the zero section of $X$ in $E$. Then zero $(s)$ has a natural structure of a $C^{\infty}$ manifold of real codimension $2 n$ in $X$, and the proper arientation class of zero $(s)$ is the Poincaré dual of $c_{n}(E)$.

Proof. Let $\gamma$ be a smooth singular $n$-cycle in the interior of $X$, which is transversal to zero $(s)$, i.e., every singular simplex $\sigma$ which intersects zero $(s)$, meets it in an isolated interior point. Just as in the proof of (6.4) one now concludes from (6.7) that

$$
\int_{\sigma} c_{n}(E)=\text { intersection }(\sigma, \operatorname{zero}(s))+\int_{\partial \sigma} s^{*} \eta(E) \text {. }
$$

Hence summing over $\sigma$ in $\gamma$, we obtain:

$$
\int_{\gamma} c_{n}(E)=\text { intersection }(\operatorname{zero}(s), \gamma) \text {. Q.E.D. }
$$

Remark $I$. It is of course artificial to bring in any assumption of complex analyticity when dealing with the Gauss-Bonnet theorem, and one could modify this account by defining $\eta$ directly on any smooth hermitian bundle. However as we are primarily interested in the complex analytic case here and the more general approach would have taken us even further afield, we only discussed that case. In the next integration the analytic structure plays a vital role.

Remark II. There are two quite straightforward generalizations of the exact sequence 


$$
0 \rightarrow S_{1}(V) \rightarrow T_{1}(V) \rightarrow Q_{1}(V) \rightarrow 0
$$

over $P_{1}(V)$, for which we will have use later on.

Namely, if $P_{n}(V)$ denotes the Grassmanian of $n$-dimensional subspaces of $V$, we have the corresponding sequence

$$
0 \rightarrow S_{n}(V) \rightarrow T_{n}(V) \rightarrow Q_{n}(V) \rightarrow 0
$$

over $P_{n}(V)$, with $T_{n}(V)=P_{n}(V) \times V$, and $S_{n}(V)$ being the subset of pairs $(A, v)$ with $v \in A$.

Finally this construction makes sense when $V$ is replaced by a vector bundle $E$ over $X$. That is, one defines $P_{n}(E)$ as the pairs $(A, x)$ consisting of a point $x \in X$, and an $n$ dimensional subspace $A$ in $E_{x}$. One lets $T_{n}(E)$ be the bundle induced from $E$ over $P_{n}(E)$ by the projection $P_{n}(E) \rightarrow X$, and then obtains an exact sequence

$$
0 \rightarrow S_{n}(E) \rightarrow T_{n}(E) \rightarrow Q_{n}(E) \rightarrow 0 \quad \text { over } P_{n}(E)
$$

where $S_{n}(E)$ consists of the triples $(A, x, e)$ with $e \subset A$.

\section{The second integration; definition of the order function}

We are now in a position to discuss the generalized first inequality of the Nevanlinna theory. Just as in Section 6 , we will be dealing with a holomorphic hermitian vector bundle $E$ over the complex manifold $X$, however instead of assuming that $X$ is compact we assume only that $X$ admits a "concave exhaustion" $f$. By definition, such an exhaustion is a smooth real valued function, $f$, on $X$ such that

(7.1) $f$ maps $X$ onto $\mathbf{R}^{+}$

(7.2) $f$ is proper, that is, $f^{-1}(K)$ is compact whenever $K$ is.

(7.3) The $(1,1)$ form $d d^{c} f$ is $\leqslant 0$ for large values of $f$.

With respect to such an exhaustion of $X$, one defines the order-function of $E$, by the formula

$$
T(r)=\int_{-\infty}^{r}\left\{\int_{X_{r}} c_{n}(E)\right\} d r ; \quad X_{r}=\{x \mid f(x) \leqslant r\} .
$$

The behavior of $T(r)$ as $r \rightarrow+\infty$ is then to be thought of as the analogue of $\int_{X} c_{n}(E)$ in the compact case.

One next defines a corresponding order function for the number of zeroes of a section $s$ on $E$ which is assumed to have only isolated zeroes, by the formula

$$
N(r, s)=\int_{-\infty}^{r} \operatorname{zero}\left(s, X_{r}\right) d r
$$

where zero $\left(s, X_{r}\right)=\sum \operatorname{zero}(s, p), p$ ranging over the zeroes of $s$ interior to $X_{r}$. 7-652932 Acta mathematica 114. Imprimé le 11 août 1965. 
We note that if the integral along the boundary of $X_{r}$ could be disregarded, the formula (6.5) would imply that $N(r, s)=T(r)$. This is of course false in general, however we do have the following estimate of this error term under certain circumstances.

FIRST MAIN THEOREM. Let $E$ be a positive Hermitian bundle over $X$ where $X$ has a concave exhaustion $f$. Let $s$ be a holomorphic section of $E$ with isolated zeroes, and let $N(r, s)$ be the order function of these zeroes. Then

$$
N(r, s)<T(r)+\text { constant }
$$

where $T(r)$ is the order function of $E$.

In particular if $c_{n}(E)>0$ at some point of $X$, then $\overline{\lim }\{N(r, s) / T(r)\} \leqslant 1$. Hence the deficiency measure of $s$, defined by: $\delta(s)=1-\lim \{N(r, s) / T(r)\}$ satisfies the inequality

$$
0 \leqslant \delta(s) \leqslant 1
$$

Proof. Let $\Gamma \subset X \times \mathbf{R}$ be the graph of $f$, and let $W$ be the region in $X \times \mathbf{R}$, which is "above" $\Gamma$ and "below" the slice $X \times r$ :

$$
W=\{(x, t) \mid f(x) \leqslant t \leqslant r ; x \in X, t \in \mathbf{R}\} .
$$

The natural projection $W \rightarrow X_{r}$ will be denoted by $\sigma$.

It is then clear that

$$
T(r)=\int_{W} \sigma^{*} c_{n}(E) d t
$$

with $W$ the orientation induced by the product orientation on $X \times \mathbf{R}$, and $d t$ the volume element on $\mathbf{R}$.

Suppose now that $s \neq 0$ on $X_{r}$. Because $|s|<1$ we may think of $s$ as a section of $B^{*}(E)$ so that on $X_{r}$

$$
c_{n}(E)=\frac{1}{4 \pi} s^{*} d d^{c} \varrho
$$

where $\varrho=\varrho(E)$ is the form given by Theorem I on $B^{*}(E)$.

We may therefore write $\sigma^{*}\left(c_{n}(E) \wedge d t\right)$ as $d\left\{\sigma^{*} s^{*} d^{c} \varrho \wedge d t\right\} / 4 \pi$ and apply Stokes' formula to obtain:

$$
T(r)=\frac{1}{4 \pi} \int_{\partial W} \sigma^{*} s^{*} d^{c} \varrho \wedge d t
$$

Now the boundary of $W$ clearly falls into the top-face $X_{r} \times r$, and the bottom face $\Gamma_{r}$, which is the graph of $f \mid X_{r}$ :

$$
\partial W=\left(X_{r} \times r\right) \cup \Gamma_{r} .
$$


Further, the integrand in (7.8) clearly restricts to zero on the top-face, as $d t$ does. Hence, keeping track of the orientation we obtain $-1 / 4 \pi \int_{\Gamma_{r}} \sigma^{*} s^{*} d^{c} \varrho \wedge d t$ for this integral, so that identifying $\Gamma_{r}$ with $X_{r}$ one obtains:

$$
T(r)=\frac{1}{4 \pi} \int_{X_{r}}-s^{*} d^{c} \varrho \wedge d f
$$

We next use the fact that $s$ is holomorphic. This implies that $s^{*} d^{c} \varrho=d^{c} s^{*} \varrho$ and, furthermore, that $s^{*} \varrho \in A^{n-1, n-1}(X)$.

Now a direct verification shows that the following identity is valid:

Proposition 7.10. If $X$ is an n-dimensional complex manifold, and $f \in A^{0}(X)$, $\lambda \in A^{n_{-1}, n_{-1}}(X)$, then

$$
d f \wedge d^{c} \lambda=d\left(d^{c} f \lambda\right)-\lambda d d^{c} f
$$

When this identity is substituted into (7.9) and the Stokes formula is used once more in the first term we obtain the relation:

$$
T(r)=\frac{1}{4 \pi} \int_{\partial x_{r}} d^{c} f \cdot \lambda-\frac{1}{4 \pi} \int_{x_{r}} \lambda d d^{c} f, \quad \lambda=s^{*} \varrho(E)
$$

and this is the basic integral relation which lies behind the first main theorem when $s$ does not vanish on $X_{r}$.

In the case when $s$ vanishes at isolated points $p_{i}, i=1, \ldots, m$, in $X_{r}$ let $X_{r}^{\varepsilon}$ be obtained from $X_{r}$ by deleting $\varepsilon$ discs $D_{i}(\varepsilon)$ about the $p_{i}$, and let $W(\varepsilon)$ be $W$ with the solid cylinders $C_{i}(\varepsilon)$ above these dises removed. Now

$$
T(r)=\frac{1}{4 \pi} \lim _{\varepsilon \rightarrow 0} \int_{W(\varepsilon)} \sigma^{*} s^{*} d d^{c} \varrho \wedge d t
$$

and on $W(\varepsilon)$ we may apply our earlier argument. However this time $\partial W(\varepsilon)$ also contains: the boundaries of the cylinders $C_{i}(\varepsilon)$, contributing the extra term

$$
\frac{1}{4 \pi} \sum \int_{\partial c_{i}(\varepsilon)} \sigma^{*} s^{*} d^{c} \varrho \wedge d t
$$

which by $(6.5)$ is easily seen to tend to $N(r, s)$ as $\varepsilon \rightarrow 0$. Hence (7.12) is modified to:

$$
T(r)-N(r, s)=\frac{1}{4 \pi} \lim _{\varepsilon \rightarrow 0}\left[\int_{\partial x_{r}^{\varepsilon}} d^{c} f \lambda-\int_{x_{r}^{\varepsilon}} \lambda d d^{c} f\right] ; \quad \lambda=s^{*} \varrho(E)
$$

We next apply the following lemma which will be proved later by an estimate. 
Lемма. In the situation just described;

(7.14) $\lim _{\varepsilon \rightarrow 0} \int_{\delta D(\varepsilon)}\left|d^{c} f \wedge \lambda\right|=0, \quad \lambda=s^{*} \varrho(E)$.

(7.15) The form $\lambda d d^{c} f$ is absolutely integrable on $X_{r}$.

(7.16) The form $d^{c} f \wedge \lambda$ is absolutely integrable on $\partial X_{r}$.

In view of this good state of affairs we may pass to the limit in (7.13) to obtain the fundamental integral formula:

$$
T(r)-N(r, s)=\frac{1}{4 \pi} \int_{\partial X_{r}} d^{c} f \wedge \lambda-\frac{1}{4 \pi} \int_{X_{r}} \lambda d d^{c} f, \quad \lambda=s^{*} \varrho(E) .
$$

The inequality of the first main theorem now follows directly. Indeed, by Theorem I, $\lambda \geqslant 0$ on $X_{r}$. By assumption $d d^{c} f \leqslant 0$ on the complement of some $X_{r_{0}}$. Hence $-\lambda d d^{c} f \geqslant 0$ there, and so the second term on the right hand side is greater than some constant.

The term $\int_{\partial x_{r}} d^{c} f \wedge \lambda$ is actually non-negative, for the following reason. Recall first that $X_{r}$ was oriented by the positive $(n, n)$-forms on $X$. Recall also that the orientation induced by the Stokes formula $\int_{X_{r}} d \omega=\int_{\partial x_{r}} i^{*} \omega$, on $\partial X_{r}$ is characterized by the condition:

A real $(2 n-1)$ form $\xi$ on $X$ restricts to a positive form on $\partial X_{r}$, relative to the induced orientation, if and only if $d f \wedge \xi$ is positive on $X$, near $X_{r}$.

Hence the sign of $\int_{\partial x_{r}} d^{c} f \wedge \lambda$ is determined by the sign of $d f \wedge d f^{c} \wedge \lambda$ on $X_{r}$. But if $f$ is any real valued function, then $d f \wedge d^{c} f \wedge \lambda$ is also positive. Q.E.D.

The inequality now follows as we have proved that $T(r)-N(r, s)>$ constant.

Proof of the lemma. We need to estimate the form $\lambda=s^{*} \underline{0}$ near an isolated singularity, $p$, of $s$. For this purpose choose a holomorphic trivialization $\varphi: E \rightarrow E_{p}$, of $E$ near $p$. Then $s^{*} \varrho$ will be close to $(\varphi \circ s)^{*} j_{p}^{*} \varrho$ near $p$, so that it is sufficient to study this form near $p$. Our first task is therefore to describe $j_{p}^{*} \varrho$.

Let $\pi: E_{p} \rightarrow p$, and set $E=\pi^{-1}\left(E_{p}\right)$ be the induced bundle over $E_{p}$. The identity map $E_{p} \rightarrow E_{p}$, then defines a section $s$ of $E$, which does not vanish on $E_{p, 0}=E_{p}-0$, and so generates a sub-bundle $E_{I}$ of $E$ there. Let $j_{p}: E_{p_{0} 0} \rightarrow E$ be the inclusion. The form $j_{p}^{*} \varrho$ is then made out of the curvature forms of $E_{I}$ and $E_{I I}=E / E_{I}$, according to the prescription (5.10). Now as $E$ is clearly the trivial Hermitian bundle over $E_{p}-0$, the curvature of $E$ vanishes identically. Hence $K\left(E_{I I}\right)$ has the form $\delta \wedge \delta^{*}$, where $\delta$ is the degree zero operator $P_{I I} D P_{I}$ of Section 4, and may be computed explicitly. Indeed let $u_{\alpha}, \alpha=1, \ldots, n$, be an orthonormal frame for $E_{p}$, and let $z_{\alpha}$ be the corresponding local coordinates on $E_{p}$ so that

$$
\sum z_{\alpha}(q) u_{\alpha}=q, \quad q \in E_{p},
$$


and let $r(q)=\left(\sum\left|z_{\alpha}(q)\right|^{2}\right)^{\frac{1}{2}}$. If we interpret the $u_{\alpha}$ as the constant sections of $E$ then the identity section $s$ is given by $s(q)=\sum z_{\alpha}(q) u_{\alpha}$, and so $D s(q)=\sum d z_{\alpha} u_{\alpha}$. It follows that at a point $q$, with $z_{1}(q)=r(q), z_{\beta}(q)=0, \beta=2, \ldots, n$, the curvature matrix relative to the frame of $E_{I I}$ determined by the $u_{\beta}, \beta=2, \ldots, n$, is simply given by

In particular then,

$$
\frac{1}{r^{2}} d z_{\alpha} \wedge d \bar{z}_{\beta}, \quad \alpha, \beta=2, \ldots, n
$$

$$
c_{n-1}\left(E_{I I}\right)=\frac{\text { const }}{r^{2(n-1)}} d z_{2} \wedge d \bar{z}_{2} \wedge \ldots \wedge d z_{n} \wedge d \bar{z}_{n}
$$

With the aid of (7.18) one may estimate all the terms of (5.10) and so conclude that:

$$
j_{p}^{*} \varrho=r^{-2(n-1)} \log r \cdot \omega_{1}+\sum_{i \geqslant 2} r^{-2 n-i} \omega_{i}
$$

where $\omega_{i}$ is bounded on all of $E_{p, 0}$.

The lemma now follows easily from (7.19).

Assume first that $s$ is transversal to the zero section at $p$. Then the Jacobian of $p \circ s$ is not zero at $p$. For our convergence questions $\varphi \circ s$ may therefore be replaced by the identity map. Now let $D(\varepsilon)$ be the ball of radius $\varepsilon$ about 0 in $\mathbf{C}_{n}$. Then if $\varrho$ is of the type given by (7.19) we clearly have

$$
\int_{\partial D(\varepsilon)} \varrho \wedge \theta \rightarrow 0, \text { and } \int_{D(\varepsilon)} \varrho \wedge \theta \wedge \bar{\varphi} \rightarrow 0
$$

for any bounded 1-forms $\theta$ and $\varphi$ because the volume of the sphere of radius $r$ is of the order $r^{2 n-1}$ and so dominates $r^{2(n-1)} \log r$. The lemma therefore is clear in that case. For a general isolated zero of $s$, there exist arbitrarily small perturbations of $s$ with only a finite number of nondegenerate zeroes near $p$. Hence our lemma also holds in that case.

\section{Equidistribution in measure}

In this section we derive the generalized first equidistibution theorem from the first main theorem with the aid of two essentially known but hard to refer to propositions which are then taken up in later sections.

We start with a statement of the theorem we are after:

EQUIDISTRIBUTION THEOREM. Let $E$ be a complex vector bundle of fiber-dimension $n$, over the complex connected manifold $X$, and let $V \subset \Gamma(E)$ be a finite dimensional space of holomorphic sections of $E$. Assume further that,

(8.1) $X$ admits a concave exhaustion $f$, in the sense of Section 7.

(8.2) $V$ is sufficiently ample in the sense that: 
ג) The map $s \rightarrow s(x)$, maps $V$ onto $E_{x}$ for each $x \in X$.

$\beta$ ) There is some $s \in V$, and some $x_{0} \in X$, so that $s: X \rightarrow E$, is transversal to the zero-section of $E$ at $x_{0}$.

Under these circumstances nearly every section in $V$ vanishes the same number of times.

Precisely, a hermitian structure on $V$ defines a hermitian structure on $E$, and hence $a$ deficiency measure $\delta(s)$ on the generic sections of $V$. The assertion is that except for a set of measure $0, \delta(s)=0$.

Proof. We first of all remark more explicitly on how the hermitian structure on $V$ defines $T(r), N(r, s)$ etc.

For this purpose let $m=\operatorname{dim} V-n$, and consider the exact sequence

$$
0 \rightarrow S_{m}(V) \rightarrow T_{m}(V) \rightarrow Q_{m}(V) \rightarrow 0 \quad \text { over } P_{m}(V) .\left(^{(1)}\right.
$$

By (8.2) part $\alpha$, the map $\varepsilon_{x}: V \rightarrow E_{x}$ which sends $s$ into $s(X)$ is onto. Hence, $k_{x}$, the kernel of $\varepsilon_{x}$ has $\operatorname{dim} m$. Now it is clear from (8.3) that the induced map $e_{V}: X \rightarrow P_{m}(V)$, defined by $x \rightarrow k_{x}$, determines an isomorphism of $Q_{m}(V)$ with $E$ : That is

$$
e_{V}^{-1}\left\{Q_{m}(V)\right\} \simeq E \text {. }
$$

A hermitian structure on $V$ induces one on $T_{m}(V)$ and hence on $Q_{m}(V)$ and $S_{m}(V)$ and hence by (8.4) also on $E$.

Note further that $Q_{m}(V)$ is positive in this structure as $T_{m}(V)$ clearly has zero curvature and "quotient bundles are always more positive" (see Section 4). Hence $E$ is also positive. Finally, the "height of a section $s$ " in $V$ at any point $x \in X$ is clearly bounded by the length of $s$ "qua element" in $V$. In short we may, after possibly multiplying $s$ by a suitable constant, not only apply the notions of Section 7 to $E$, but we also obtain the inequality of the first main theorem:

$$
N(r, s)<T(r)+\text { constant }
$$

valid for sections with isolated signularities.

Now condition $\beta$, of (8.2) is seen to imply by an explicit check, that $e_{V}^{*} c_{n}\left\{Q_{n}(V)\right\}$ is strictly positive near $x_{0}$ (see remark at end of Section 9). Hence $T(r) \rightarrow+\infty$, so that (8.4) implies the inequality:

$$
0 \leqslant \varlimsup \frac{N(r, s)}{T(r)} \leqslant 1
$$

We now need the following two propositions:

Proposition 8.6. Under the assumption (8.2) nearly all $s \subset V$, have only isolated zeroes. In fact nearly all sections $s \in V$ are transversal to the zero section of $E$.

(1) See the remark at the end of Section 6 . 
Proposition 8.7. Under the assumptions (8.2) we have the equality

$$
\int_{P_{1}(V)} N(r, s) \omega=T(r), \quad s \in[s] \in P_{\mathbf{1}}(V)
$$

where $\omega$ is the volume on $P_{1}(V)$ invariant under the group of isometries of $V$ and normalized by $\int_{P_{1}(v)} \omega=1$; while $N(r, s)$ is the order function interpreted as a function on $P_{1}(V)$.

The equidistribution theorem: $\delta(s)=0$ almost everywhere now follows directly. Indeed by $(8.5) 0 \leqslant \delta(s) \leqslant 1$. Hence $\int_{P_{1}(V)} \delta(s) \omega \geqslant 0$. On the other hand by (8.7) we have

$$
\varlimsup \int_{P_{1}(V)}\{N(r, s) / T(r)\} \omega=1 \text {, whence } \int_{P_{1}(V)} \varlimsup \lim \{N(r, s) / T(r)\} \omega \geqslant 1 \text {, }
$$

and so finally

$$
\int_{P_{1}(V)} \delta(s) \omega \geqslant 0 . \quad \text { Q.E.D. }
$$

\section{The proof of Proposition 8.6}

This assertion is clearly a variant of Bertini's theorem, and is proved along the same lines. Briefly the argument runs as follows.

Let $K=e_{V}^{-1}\left\{S_{m}(V)\right\}$, and consider the associated projective bundle $P_{1}(K)$ over $X$. (See the remark at the end of Section 6 for these concepts.) There is a natural imbedding of $P_{1}(K)$ in $X \times P_{1}(V)$ as the subset:

$$
P_{1}(K)=\left\{(x, l) \text { with } l \subset k_{x}\right\}
$$

and we let $\pi: P_{1}(K) \rightarrow P_{1}(V)$ be the projection on the second factor. Next let $\Sigma \subset P_{1}(K)$ be the subset of those pairs $(x, l)$ for which $l$ is generated by a section $s: X \rightarrow E$, which is not transversal to the zero section at $x$.

This is the singular set in $P_{1}(K)$, and it is clear that the complement in $P_{1}(V)$ of the image of $\Sigma$ under $\pi$ consists of transversal sections. Now $\operatorname{dim} P_{1}(K)=\operatorname{dim} P_{1}(V)$ because $\operatorname{dim} X$ equals the fiber-dimension of $E$. Hence if we can show that the codimension of $\Sigma$ in $P_{1}(K)$ is $\geqslant 1$, then $\Sigma$ and $\pi(\Sigma)$ will have measure zero and the proposition will be established.

Our aim is therefore to show that $\Sigma$ is the zero set of a not identically zero section of a certain line-bundle over $P_{1}(K)$. To see this, remark first that if $s \in k_{x}$ and if $U \in X_{x}$ is a tangent vector to $X$ at $x$, then the derivative of $s$ in the direction $U$, is a well determined element $U \cdot s$ of $E_{x}$. (Recall that $s \in k_{x} \Leftrightarrow s(x)=0$; to differentiate general sections one of course needs a connection, however at the zeroes of $s$ all connections on $E$ define the same derivative.) 
This operation therefore leads to a map

$$
J^{t}: k_{x} \rightarrow \operatorname{Hom}\left(X_{x}, E_{x}\right)
$$

and it is easy to see that $\Sigma=\left\{(x, l) \in P_{1}(K) \mid \operatorname{det} J^{t}(s)=0,[s]=l\right\}$.

Now when lifted to $P_{1}(K)$, det $J^{t}$ may be interpreted as a section of the line bundle

$$
L=\operatorname{Hom}\left\{S(K), \operatorname{Hom}\left(\Lambda^{n} T, \Lambda^{n} E\right\}\right.
$$

where $\Lambda^{n}$ denotes the $n$th exterior power and $T$ denotes the tangent bundle of $X$ lifted to $P_{1}(K)$. Thus $\Sigma=$ zero set of $\operatorname{det} J^{t} \in \Gamma(L)$. On the other hand condition (8.2) $\beta$ ) demands precisely, that det $J^{t}$ be non-zero at some point of $P_{1}(K)$. Because $X$ is connected it follows that $\operatorname{codim} \Sigma=1$. Q.E.D.

Remark. The transpose of $J^{t}$ is given by

$$
J: X_{x} \rightarrow \operatorname{Hom}\left(k_{x}, E_{x}\right)
$$

and may be identified with the Jacobian of $e_{V}$ at $x$. Thus condition (8.2) $\beta$ ) implies that $e_{V}$ is an immersion near $x_{0}$. From this it follows easily that $c_{n}(E)>0$ near $x_{0}$.

\section{Some remarks on integral geometry. The proof of Proposition 8.7}

Suppose $\pi: Y \rightarrow X$ is a smooth fibering of compact manifolds with oriented fiber $F$. In that situation there is a well-defined operation

$$
\pi_{*}: A^{k}(Y) \rightarrow A^{k-f}(X), \quad f=\operatorname{dim} F
$$

called integration over the fiber, which "realizes" the adjoint of $\pi^{*}$ in the sense that if $X$ and $Y$ are oriented compatibly then for any $\varphi \in A(X), \psi \in A(Y)$ we have the identity:

$$
\int_{Y} \psi \pi^{*} \varphi=\int_{X}\left(\pi_{*} \psi\right) \cdot \varphi
$$

The existence of $\pi_{*}$ on the "form level", suggests the following definition.

DEFINITION 10.2. Let $\varphi \in A^{k}(X)$. By an integral representation of $\varphi$ we mean a triple, $(Y, Z, \omega)$ where $Y_{\rightarrow}^{\rightarrow} X$ is an oriented fibering over $X$, with projection $\pi$, and $\omega \in A^{m}(Z)$ is a volume element $\left.{ }^{1}\right)$ on the oriented m-manifold $Z$, together with a map $\sigma: Y \rightarrow Z$, such that

$$
\varphi=\pi_{*} \circ \sigma^{*} \omega
$$

In general the question whether a given closed form $\varphi$ on $X$ admits an integral repre-

(1) Volume element means a nonvanishing form of top dimernsion, in the orientation class. 
sentation seems quite difficult. Certainly $\varphi$ must have integral periods and there are most probably much more subtle conditions which also have to be satisfied. For our purposes it will however be sufficient to show that the characteristic class $c_{n}(E)$ of a hermitian bundle which is ample in the sense of $(9.2) \alpha$ ) always has an integral representation. Note that if $\varphi$ has an integral representation, then any pull-back $f^{*} \varphi$ also has an integral representation. Hence it will be sufficient to get a representation theorem for $c_{n}\left[Q_{n}(V)\right]$ over $P_{n}(V)$.

In the next proposition we describe a quite general representation theorem for the Grassmann-varieties $P_{n}(V)$. We will first simplify the notation as follows: $V$ will denote a fixed hermitian vector space of dimension $d$; and we write simply $P_{n}, Q_{n}$ etc., for $P_{n}(V)$, $Q_{n}(V)$ etc. The bundle $Q_{n}$ is always considered in the hermitian structure induced on $Q_{n}$ by the trivial structure on $T_{n}$; so that the Chern forms $c\left(Q_{n}\right)$ are well-defined.

Now let $0<n<m<d$ be two integers and define $P_{n, m}=P_{n, m}(V)$ as the "flagmanifold" of pairs $\left(A_{n} \subset B_{m}\right)$ of subspaces of dimension $n$ and $m$ respectively in $V$. Let $P_{n, m} \stackrel{\sigma}{\rightarrow} P_{n}$, and $P_{n, m} \stackrel{\pi}{\rightarrow} P_{m}$ be the natural projections, $\sigma(A, B)=A ; \pi(A, B)=B$, and consider the diagram:

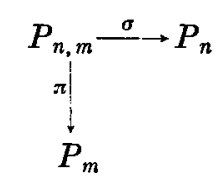

We then have the following proposition.

REPRESENTATION THEOREM. In the diagram (10.4) one has the relation:

$$
c_{d-m}\left\{Q_{m}\right\}=\pi_{*} \cdot \sigma^{*} c_{d-n}\left\{Q_{n}\right\}
$$

Proof. One may of course compute everything explicitly in these examples and so verify (10.5). There is also a much simpler global proof based on the corollary (6.13). The argument runs as follows.

Consider the action of the group of isometries of $V$, say $I(V)$, on $P_{n}$. From the rather canonical definition of the bundles $Q_{n}$ it is then not hard to see that their Chern forms $c\left\{Q_{n}\right\}$, are invariant under $I(V)$.

It is also easy to see that $P_{n}$ is a symmetric space of $I(V)$ whence every real cohomology class of $P_{n}$ contains a single invariant differential form. We may therefore prove (10.5) by checking it on the cohomology level. Alternately we may pass to homology by Poincare duality. Then $\sigma^{*}$ corresponds simply to inverse image of the dual cycle to $c_{v-n}\left\{Q_{n}\right\}$ and $\pi_{*}$ corresponds to projection. 
Now let $v$ be a non-zero element of $V$, and consider the section $s_{v}$ it determines in $Q_{n}$ over $P_{n}$. This is clearly a transversal section and we have: zero $\left(s_{v}\right)=\left\{A_{n} \supset v\right\}$. Similarly $v$ determines the section $s_{v}^{\prime}$ of $Q_{m}$ over $P_{m}$, and, zero $\left(s_{v}^{\prime}\right)=\left(B_{m} \supset v\right)$. Thus we get the formula:

$$
\operatorname{zero}\left(s_{v}^{\prime}\right)=\pi \circ \sigma^{-1} \text { ozero }\left(s_{v}\right) \text {. }
$$

Finally, by Proposition 6.13 these zero-sets are duals of the corresponding Chern classes, so that (10.6) proves (10.5) on the homology level. (Because our sections are holomorphic there is no problem with orientations.)

We discuss next, the geometric implications of an integral representation. Consider then the diagram:

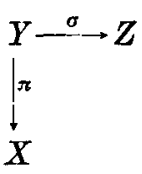

with $\varphi=\pi_{*} \circ \sigma^{*} \omega$. If $z$ is not in the critical set $\sigma$ (a point $p$ is critical if at some point of $\sigma^{-1}(p)$, the differential $d \sigma$ is not onto its tangent space $\left.Z_{p}\right)$. Then $\sigma^{-1}(z)$ is a well-defined oriented manifold, so that the pair $\left(\sigma^{-1}(z) ; \pi\right)$ determines a smooth oriented singular submanifold $X$ which we denote by $c(z)$. As already remarked, each $c(z)$ represents the homology class dual to $\varphi$. However, we have more than that; the family $c(z)$ determines not only the dual homology class of $\varphi$ but also the value of $\varphi$ on any singular $k$-submanifolds $f: K \rightarrow X$.

Proposition 10.8. Let the $k$-form $\varphi \in A^{k}(X)$ have an integral representation $\omega$ on $Z$, in the sense of (10.1). Then for any compact singular submanifold $f: K \rightarrow X$ of dimension $k$; one has:

(10.9) The intersection $n(K, c(z))$ of $K$ with $c(z)$ is well-defined except for a set of measure zero in $Z$.

$$
\int_{K} f^{*} \varphi=\int_{z} n(K, c(z)) \omega
$$

Outline of Proof. Let $Y(f)=f^{-1}(Y)$ be the bundle induced by $Y$ over $K$, under $f$, and let $f^{\prime}: Y(f) \rightarrow Y$ be the bundle map covering $f$. If $\pi_{K}: Y(f) \rightarrow K$ is the projection we have:

$$
\int_{K} f^{*} \varphi=\int_{K} \pi_{K^{*}} f^{\prime *} \circ \sigma^{*} \cdot \omega=\int_{Y(f)}\left(\sigma \circ f^{\prime}\right)^{*} \omega
$$

where the first step follows from the identity $f^{*} \circ \pi^{*}=\pi_{K}^{*} \circ f^{* *}$ and the second one from the adjoint property (10.1) of $\pi_{*}$.

One next considers the map $\lambda=\sigma \circ f^{\prime}: Y(f) \rightarrow Z$. A count of dimension shows that 
$\operatorname{dim} Y(f)=\operatorname{dim} Z$. Hence on the complement of the critical value set of $\lambda$ the degree of $\lambda$ at $z$, is well-determined and computes the algebraic number of sheets with which some vicinity of $z$ is covered. Thus

$$
\int_{Y(f)} \lambda^{*} \omega=\int_{Z} \operatorname{deg}(\lambda ; z) \omega .
$$

Finally consider the points of $\lambda^{-1}(z)$, with $z$ a regular (i.e., not critical) value of $\lambda$. We see first of all that these points correspond precisely to the intersections of $c(z)$ with $f(K)$ and furthermore that all these intersections are transversal so that the intersection numbers are well-defined and their algebraic sum, is precisely $\operatorname{deg}(\lambda, z)$. The theorem now follows from the fact that the critical set of smooth maps have measure zero.

The proposition (8.7) which motivated this excursion is a direct consequence of the formulae (10.5) and (10.10). Indeed, let $r=d-n$, and consider the exact sequence

$$
0 \rightarrow S_{r}(V) \rightarrow T_{r}(V) \rightarrow Q_{r}(V) \rightarrow 0 \quad \text { over } P_{r}(V)
$$

Let $e_{V}: X \rightarrow P_{r}(V)$ be the evaluation map, so that $e_{V}^{-1}\left[Q_{r}(V)\right]=E$. We now apply (10.5) with $n=1$, and $m=r$. Thus the diagram we need is<smiles>[Te][Te]</smiles>

Applying (10.5) one obtains $c_{n}\left[Q_{r}\right]=\pi_{*} \circ \varphi^{*} c_{d-1}\left\{Q_{1}\right\}$ and it is clear that $c_{d-1}\left(Q_{1}\right)$ is a volume of measure 1 on $P_{r}(V)$-because $s_{v}$ vanishes at a single point for instance! Now one applies (10.8) with $X_{r}$ replacing $K$, and $e_{V}$ replacing $f$, to obtain:

$$
\int_{X} c_{n}(E)=\int_{P_{1}(V)} n(r, s) \omega
$$

with $n(r, s)$ simply the number of zeroes of $s \in V$ on $X_{r}$ ( $s$ a generic section). Integrating with respect to $r$, we conclude that

$$
T(r)=\int_{P_{1}(V)} N(r, s) \omega . \quad \text { Q.E.D. }
$$

\section{The Nevanlinna Theorem}

We conclude this paper with a short account of the classical Nevanlinna theorem. In particular we would like to show that the second main theorem of the Nevanlinna theory is also a consequence of the integral formula which yields the first main inequality. 
We therefore specialize all our constructions as follows:

(11.1) For $X$ we take the plane $C$, with an exhaustion, $f(z)$, for which $f(z)=\log |z|$ when $|z| \geqslant 1$.

(11.2) $E$ is the trivial line bundle over $X$, so that $\Gamma(E)$ is the space of holomorphic functions on $\mathbf{C}$.

(11.3) $V \subset \Gamma(E)$ is a 2-dimensional sufficiently ample subspace of $\Gamma(E)$, i.e., one generated by two functions $s_{1}$ and $s_{2} \in \Gamma(E)$ which are not proportional, and which do not have any common zeroes.

Remarks. $\alpha$ ) The assumption that $E$ is the trivial bundle is really no restriction as all holomorphic bundles over $\mathbf{C}$ are known to be trivial.

B) The function $\log |z|$, is harmonic for large $|z|$ and therefore has the property $d d^{c} f=0$. Hence $f$ does define a "concave" exhaustion. On the other hand, the function $|z|^{2}$ would not do, because

$$
d d^{c}|z|^{2}=2 i d z \wedge d \bar{z} \geqslant 0 .
$$

$\gamma)$ By (11.3) every $s \in V$ is of the form $a s_{1}+b s_{2}$. Hence the zeroes of $s$ correspond to the points where $r(z)=s_{1}(z) / s_{2}(z)=-b / a$. In short we are dealing precisely with the value distribution of the meromorphic function $r(z)$.

The refinement of the equidistribution theory which is possible in this situation is in the first place a consequence of the fact that our exhaustion function is harmonic, so that $d d^{c} f=0$ for large values of $|z|$. It follows that (7.16) specializes to the formula:

$$
T(r)-N(r, s)=\frac{1}{4 \pi} \int_{\partial x_{r}} \log \left\{1 /|s|^{2}\right\} \cdot d^{c} f+\text { const. }
$$

Indeed, in the case of line bundles, the form $\varrho$ of Theorem I may simply be taken to be $\log (1 / N(s))$. The formula (11.4) is furthermore valid for all $s \in V-0$, because all these sections vanish at isolated points.

Consider then a set of $q$ sections $s_{i} \in V$, no two of which are dependent. Our aim is the Nevanlinna inequality:

$$
\sum_{i=1}^{q} \delta\left(s_{i}\right) \leqslant 2
$$

where

$$
\delta\left(s_{i}\right)=1-\overline{\lim } \frac{N\left(r, s_{i}\right)}{T(r)} .
$$

For this purpose observe first of all that a repeated application of (11.4) yields,

$$
q T(r)-\sum N\left(r, s_{i}\right)=\frac{1}{4 \pi} \int_{\partial x_{r}} \log \mu^{2} \cdot d^{c} f+\text { const }
$$


with

$$
\mu^{2}=\left\{\prod_{1 \leqslant i_{q}}\left|s_{i}\right|^{2}\right\}^{-1}
$$

Hence if $M(r)$ denotes the term $1 / 4 \pi \int_{\partial x_{r}} \log \mu^{2} d^{c} f$, then we need to prove the inequality:

$$
\underline{\lim } \frac{M(r)}{T(r)} \leqslant 2
$$

to establish (11.5).

The estimation of $M(r)$ proceeds by first correcting $M(r)$ for the singular points of our evaluation map

$$
e_{V}: X \rightarrow P_{1}(V)
$$

Let $\tau(X)$ and $\tau\left[P_{1}(V)\right]$ be the respective holomorphic tangent bundles of $X$ and $P_{1}(V)$, so that $d e_{V}$ becomes a section of the line bundle:

$$
\operatorname{Hom}\left[\tau(X), e_{V}^{-1} \cdot \tau\left[P_{1}(V)\right]\right] \text {. }
$$

We consider the global section $\partial / \partial z$ of $X$, and set $t=d e_{V}(\partial / \partial z)=d e_{V} / d z$. Then $t$ is a holomorphic section of $L=e_{V}^{-1} \tau\left[P_{1}(V)\right]$ and the singular points of $e_{V}$ are precisely the zeroes of $t$.

Now the fixed Hermitian structure on $V$ which underlies all our constructions, induces a Hermitian structure on $\tau\left\{P_{1}(V)\right\}$ through the well known isomorphism:

$$
\tau\left[P_{n}(V)\right]=\operatorname{Hom}\left[S_{n}(V), Q_{n}(V)\right] .
$$

One may therefore apply the first main integral formula to the section, $t$, of $L=e_{V}^{-1} \circ \tau\left[P_{1}(V)\right]$, and so obtains:

$$
\int_{-\infty}^{r} d r \int_{X_{r}} c_{1}(L)-N(r, t)=\frac{1}{4 \pi} \int_{\partial x_{r}} \log 1 /|t|^{2} \cdot d^{c} f+\text { const. }
$$

Once one identifies the first integral in (11.12) with $2 T(r)$, this formula becomes the socalled second main theorem of the Nevanlinna theory. Actually, that identification follows directly from the following quite general proposition:

PROPOSITION 11.13. In the natural Hermitian structure on $\tau\left\{P_{n}(V)\right\}$ induced by (11.11), one has the identity of Chern forms:

$$
c_{1}\left\{\tau\left[P_{n}(V)\right]\right\}=\operatorname{dim} V \cdot c_{1}\left[Q_{n}(V)\right] .
$$

We bring only the proof for $n=1$, the general case being similar but involving some complicated identities about the determinant. Let then $n$ be 1 , and consider the sequence (see Section 6) of bundles:

$$
0 \rightarrow S_{1} \rightarrow T_{1} \rightarrow Q_{1} \rightarrow 0
$$


over $P_{1}(V)$. Taking $\operatorname{Hom}(S, \cdot)$ of this sequence we get

$$
0 \rightarrow 1 \rightarrow \operatorname{Hom}\left(S_{1}, T_{1}\right) \rightarrow \tau\left\{P_{1}(V)\right\} \rightarrow 0 .
$$

Now we again use the Whitney formula on the homology level, and invariance under the isometries of $V$ to deduce a relation on the form-level. Namely, from (11.6)

$$
c_{1}\left\{\operatorname{Hom}\left(S_{1}, T_{1}\right)\right\}=c_{1}\left\{\tau\left[P_{1}(V)\right]\right\},
$$

while from (11.15), $c_{1}\left(S_{1}\right)=-c_{1}\left(Q_{1}\right)$ and hence $c_{1}\left\{\operatorname{Hom}\left(S_{1}, T_{1}\right)\right\}=\operatorname{dim} V \cdot c_{1}\left(Q_{1}\right)$ as was to be shown.

The formula (11.12) may therefore be used to give the following estimate:

$$
2 T(r) \geqslant \frac{1}{4 \pi} \int_{\partial X_{r}} \log 1 /|t|^{2} d^{c} f+\text { const }
$$

and this is now precisely the second fundamental inequality of the subject.

The proof of (11.8) now proceeds as follows:

Choose $0<\lambda<1$ and write:

$$
\lambda M(r)=\frac{1}{4 \pi} \int_{\partial x_{r}} \log \left\{\mu^{2 \lambda}|t|^{2}\right\} d^{c} f+\frac{1}{4 \pi} \int_{\partial X_{r}} \log \left\{1 /|t|^{2}\right\} d^{c} f .
$$

Then if the two expressions on the right are denoted by $A(r)$ and $B(r)$ respectively, we get

$$
\lambda \underline{\lim } M(r) / T(r) \leqslant \underline{\lim } A(r) / T(r)+\varlimsup \lim B(r) / T(r),
$$

whence by the second main theorem,

$$
\lambda \underline{\lim } M(r) / T(r) \leqslant \underline{\lim } A(r) / T(r)+2 .
$$

Hence if it can be shown that $\lim A(r) / T(r)=0$ for every $0<\lambda<1$ we will be done.

For this estimate, one first uses the concavity of the logarithm: Namely, if $z=r e^{i \theta}$ are the usual coordinates in $\mathbf{C}$, then for $r$ large enough; $r \geqslant r_{0}$; we have $f=l n r$, whence $d^{c} f=d \theta$ and $d f=d r / r$. In particular, $\frac{1}{2} \pi \int_{\partial x_{c}} d^{c} f=1$. It follows from the concavity of log that for $c>l n r_{0}$

$$
A(c) \leqslant \log \frac{1}{2 \pi} \int_{\partial x_{c}}|u|^{2 \lambda \mid}|t|^{2} d \theta
$$

or equivalently:

$$
e^{A(c)} \leqslant \frac{1}{2 \pi} \int_{\partial x_{c}} u^{2 \lambda}|t|^{2} d \theta
$$

This last relation is now exploited to construct an integral inequality which in some sense relates $e^{A(c)}$ with $T(c)$. For this purpose one needs the following identity, which relates $|t|^{2}$ to the Chern-form $c_{1}(E)$ which occurs in the definition of $T(c)$. The relation in question is the following one: 


$$
c_{1}(E)=\frac{1}{\pi} \cdot|t|^{2} r d r d \theta
$$

To see this formula, consider a point $x \in \mathbf{C}$, and choose a section $s_{1}$ in $V$, with $\left|s_{1}\right|=1$, and $s_{1}(x)=0$. Thus $s_{1}$ spans $k_{x}$ and is of unit length. Choose $s_{2}$ to be orthogonal to $s_{1}$ and also of unit length. Then near $x$ there is a well determined holomorphic function $\alpha(z)$ such that $s_{1}(z)-\alpha(z) s_{2}(z)$ generates $k_{1}$ for $z$ near $x$. It follows immediately from the formulae of Section 5 that in terms of this $\alpha$,

$$
c_{1}(E)=\frac{i}{2 \pi} \cdot\left|\frac{d \alpha}{d z}\right|^{2} d z \wedge d \bar{z} .
$$

Finally noting that $|d \alpha / d z|^{2}=|t|^{2}$, and that $i d z \wedge d \bar{z}=2 r d r d \theta$ one obtains (11.19).

Integrating with respect to $c$, one now deduces from (11.18) that

and so finally that

$$
\int_{c_{0}}^{c} e^{[2 c+A(c)]} d c \leqslant \frac{1}{2} \int_{x_{c}-x_{c_{0}}} c_{1}(E) \mu^{2 \lambda}
$$

$$
\int_{c_{0}}^{c} d c \int_{c_{0}}^{c} e^{[2 c+A(c)]} d c \leqslant \frac{1}{2} \int_{c_{0}}^{c} d c \int_{x_{c}-x_{c_{0}}} c_{1}(E) \mu^{2 \lambda} .
$$

The concluding steps of the proof are now expressed by the following two lemmas:

LEM A 11.22. In the notation used above,

$$
\int_{c_{0}}^{c} d c \int_{X_{c}-X_{c_{0}}} c_{1}(E) \mu^{2 \lambda}<K_{1} T(c)+K_{2}
$$

where the $K_{i}$ are constants.

Lем ма 11.23. The integral inequality

implies that the inequality

$$
\int_{c_{0}}^{c} d c \int_{c_{0}}^{c} e^{[2 c+A(c)]} d c \leqslant K_{1} T(c)+K_{2}
$$

$$
2 c+A(c) \leqslant k^{2} \log \left[K_{1}\{T(c)\}+K_{2}\right], \quad k>1,
$$

hold for arbitrarily large values of $c$.

We can clearly conclude from (11.21) and these two lemmas that $\lim A(c) / T(c)=0$, so that the Nevanlinna theorem is a direct consequence of (11.22) and (11.23).

Both Lemmas are well-known, see for instance [2]. The first one follows from an integral geometry argument, while the second one is a purely real variable inequality. 


\section{References}

This paper links classical function theory with differential geometry; it is difficult to give an adequate bibliography satisfactory to readers from both fields. We will restrict ourselves in giving some standard literature from which other references can be found:

[1]. Classical value distribution theory:

Nevanlinna, R., Eindeutige analytische Funltionen. Berlin, 1936.

[2]. Holomorphic curves in projective space:

AHLFons, L., The theory of meromorphic curves. Acta Soc. Sci. Fenn., Ser. A. 3, no. 4 (1941).

WeYL, H., Meromorphic Functions and Analytic Curves. Princeton, 1943.

[3]. Differential geometry of connections:

Kobayasin, S. \& Nomizo, K., Foundations of differential geometry. Interscience, 1963.

[4]. Characteristic classes:

HIRZeBRUCH, F., Neue topologische Methoden in der algebraischen Geometrie. Springer 1962.

[5]. Curvature of connections and characteristic classes:

Cherr, S., Differential geometry of fiber bundles. Proc. International Congress 1950, 2 (1952) 397-411.

GrIFfITHS, P. A., On a theorem of Chern. Illinois J. Math., 6, 468-479 (1962).

Received August 17, 1964 